\title{
ON ENUMERATORS OF SMIRNOV WORDS BY DESCENTS AND CYCLIC DESCENTS
}

\author{
BRITTNEY ELLZEY AND MICHELLE L. WACHS ${ }^{2}$
}

Dedicated to the memory of Jeff Remmel

\begin{abstract}
A Smirnov word is a word over the positive integers in which adjacent letters must be different. A symmetric function enumerating these words by descent number arose in the work of Shareshian and the second named author on $q$-Eulerian polynomials, where a $t$-analog of a formula of Carlitz, Scoville, and Vaughan for enumerating Smirnov words is proved. A symmetric function enumerating a circular version of these words by cyclic descent number arose in the work of the first named author on chromatic quasisymmetric functions of directed graphs, where a $t$-analog of a formula of Stanley for enumerating circular Smirnov words is proved.

In this paper we obtain new $t$-analogs of the Carlitz-ScovilleVaughan formula and the Stanley formula in which the roles of descent number and cyclic descent number are switched. These formulas show that the Smirnov word enumerators are polynomials in $t$ whose coefficients are e-positive symmetric functions. We also obtain expansions in the power sum basis and the fundamental quasisymmetric function basis, complementing earlier results of Shareshian and the authors.

Our work relies on studying refinements of the Smirnov word enumerators that count certain restricted classes of Smirnov words by descent number. Applications to variations of $q$-Eulerian polynomials and to the chromatic quasisymmetric functions introduced by Shareshian and the second named author are also presented.
\end{abstract}

Date: Nov. 29, 2018; revised April 26, 2019.

2010 Mathematics Subject Classification. 05E05, 05A05, 05A15, 05A30.

${ }^{2}$ Supported in part by NSF Grant DMS 1502606. 


\section{Contents}

1. Introduction 2

2. Expansion in the elementary symmetric functions 7

3. Chromatic quasisymmetric function of the cycle 15

4. Expansion in the power sum symmetric functions 19

5. Expansion in the fundamental quasisymmetric functions 26

6. Variations of $q$-Eulerian polynomials $\quad 32$

References 36

\section{INTRODUCTION}

We consider words $w=w_{1} w_{2} \ldots w_{n}$ over the alphabet of positive integers $\mathbb{P}$ with no adjacent repeated letters; that is $w_{i} \neq w_{i+1}$ for all $i \in[n-1]:=\{1, \ldots, n-1\}$. We refer to these words as Smirnov words as is often done in the literature; see e.g. [15, 12, 20, 3, 22, 13, 23.

For $n \geq 1$, let $W_{n}$ be the set of Smirnov words of length $n$. Now define the Smirnov word enumerator

$$
W_{n}(\mathbf{x}):=\sum_{w \in W_{n}} x_{w}
$$

where $\mathbf{x}:=x_{1}, x_{2}, \ldots$ is a sequence of indeterminates and $x_{w}:=$ $x_{w_{1}} x_{w_{2}} \cdots x_{w_{n}}$. Clearly $W_{n}(\mathbf{x})$ is a symmetric function. Carlitz, Scoville, and Vaughan [6, equation (7.12)] derived the generating function formula

$$
\sum_{n \geq 1} W_{n}(\mathbf{x}) z^{n}=\frac{\sum_{i \geq 1} i e_{i}(\mathbf{x}) z^{i}}{1-\sum_{i \geq 2}(i-1) e_{i}(\mathbf{x}) z^{i}},
$$

where $e_{i}(\mathbf{x})$ is the elementary symmetric function of degree $i$. An important consequence of this formula is that $W_{n}(\mathbf{x})$ is $e$-positive, which means that when expanded in the elementary symmetric function basis for the ring of symmetric functions, the coefficients are nonnegative.

The symmetric function $W_{n}(\mathbf{x})$ was also considered by Stanley [32] in the context of chromatic symmetric functions and by Dollhopf, Goulden, and Greene 8] in the context of pair avoiding word enumerators. Stanley also considered a circular version of Smirnov words, that is Smirnov words whose first and last letter are different. Let

$$
W_{n}^{\neq}(\mathbf{x}):=\sum_{\substack{w \in W_{n} \\ w_{1} \neq w_{n}}} x_{w}
$$


Stanley [32, Proposition 5.4] proved

$$
\sum_{n \geq 1} W_{n}^{\neq}(\mathbf{x}) z^{n}=\frac{\sum_{i \geq 1} i(i-1) e_{i}(\mathbf{x}) z^{i}}{1-\sum_{i \geq 2}(i-1) e_{i}(\mathbf{x}) z^{i}} .
$$

It follows from this formula that $W_{n}^{\neq}(\mathbf{x})$ is $e$-positive.

Given any word $w \in \mathbb{P}^{n}$, where $n \geq 1$, the descent number of $w$ is defined by

$$
\operatorname{des}(w):=\left|\left\{i \in[n-1]: w_{i}>w_{i+1}\right\}\right|
$$

and the cyclic descent number is defined by

$$
\operatorname{cdes}(w):=\left|\left\{i \in[n]: w_{i}>w_{i+1}\right\}\right|,
$$

where $w_{n+1}:=w_{1}$. Now define the refined Smirnov word enumerators

$$
\begin{aligned}
& W_{n}(\mathbf{x}, t):=\sum_{w \in W_{n}} t^{\operatorname{des}(w)} x_{w} \\
& \tilde{W}_{n}(\mathbf{x}, t):=\sum_{w \in W_{n}} t^{\operatorname{cdes}(w)} x_{w} \\
& W_{n}^{\neq}(\mathbf{x}, t):=\sum_{\substack{w \in W_{n} \\
w_{1} \neq w_{n}}} t^{\operatorname{des}(w)} x_{w} \\
& \tilde{W}_{n}^{\neq}(\mathbf{x}, t):=\sum_{\substack{w \in W_{n} \\
w_{1} \neq w_{n}}} t^{\operatorname{cdes}(w)} x_{w} .
\end{aligned}
$$

The first and fourth of these Smirnov word enumerators have been studied before. The main objective of this paper is to study the other two Smirnov word enumerators. We start with a brief review of what is known for $W_{n}(\mathbf{x}, t)$ and $\tilde{W}_{n}^{\neq}(\mathbf{x}, t)$.

1.1. Summary of known results. The refined Smirnov word enumerator $W_{n}(\mathbf{x}, t)$ arose in the work of Shareshian and the second named author on $q$-Eulerian polynomials [26]. Stanley (personal communication) observed that the $r=1$ case of [26, Theorem 1.2] is equivalent to the following $t$-analog of (1.1),

$$
\sum_{n \geq 1} W_{n}(\mathbf{x}, t) z^{n}=\frac{\sum_{i \geq 1}[i]_{t} e_{i}(\mathbf{x}) z^{i}}{1-\sum_{i \geq 2} t[i-1]_{t} e_{i}(\mathbf{x}) z^{i}},
$$

where

$$
[n]_{t}:=1+t+\cdots+t^{n-1}=\frac{t^{n}-1}{t-1} .
$$

Indeed, this follows from [26, Equation (7.7) and Theorem 3.6]; see [30, Section 4.1]. (For another proof of (1.4), see Remark 5.6.) It follows 
from (1.4) that $W_{n}(\mathbf{x}, t)$ is $e$-positive, that is, $W_{n}(\mathbf{x}, t)$ is a polynomial in $t$ whose coefficients are $e$-positive symmetric functions.

Equation (1.4) can be restated as

$$
1+\sum_{n \geq 1} W_{n}(\mathbf{x}, t) z^{n}=\frac{(1-t) E(z)}{E(t z)-t E(z)},
$$

where

$$
E(z):=\sum_{n \geq 0} e_{n}(\mathbf{x}) z^{n}
$$

The expression on the right hand side of (1.5) (or its image under the involution $\omega$ that takes $e_{n}(\mathbf{x})$ to the complete homogeneous symmetric function $\left.h_{n}(\mathbf{x})\right)$ has arisen in various other contexts. Stanley obtained the expression when considering the representation of the symmetric group on the cohomology of the toric variety associated with the dual permutohedron [31]. A conjecture of Shareshian and the second named author [28, 29, 30], proved by Brosnan and Chow [4] and subsequently by Guay-Paquet[18, generalizes the connection between the Smirnov word enumerator and the toric varieties to a connection between chromatic quasisymmetric functions and Hessenberg varieties. The expression also arose in the work of Shareshian and the second named author on the representation of the symmetric group on homology of Rees products of posets [27].

By combining (1.5) with a result of Stembridge [35], one obtains an expansion of $\omega W_{n}(\mathbf{x}, t)$ in the power sum symmetric functions $p_{\lambda}(\mathbf{x})$. The expansion is given by

$$
\omega W_{n}(\mathbf{x}, t)=\sum_{\lambda \vdash n}\left(A_{l(\lambda)}(t) \prod_{i=1}^{l(\lambda)}\left[\lambda_{i}\right]_{t}\right) \frac{p_{\lambda}(\mathbf{x})}{z_{\lambda}},
$$

where $A_{m}(t)$ is the $m$ th Eulerian polynomial, $z_{\lambda}$ is a constant associated with the partition $\lambda=\left(\lambda_{1} \geq \lambda_{2} \geq \cdots \geq \lambda_{l(\lambda)}\right)$, and $l(\lambda)$ is the length of $\lambda$.

Recall that the Eulerian polynomials $A_{n}(t)$ have two well-known combinatorial interpretations, which are given by

$$
A_{n}(t)=\sum_{\sigma \in \mathfrak{S}_{n}} t^{\operatorname{des}(\sigma)}=\sum_{\sigma \in \mathfrak{S}_{n}} t^{\operatorname{exc}(\sigma)}
$$

where $\mathfrak{S}_{n}$ is the symmetric group on $[n]$, and des and exc are MacMahon's classical equidistributed permutation statistics, descent number and excedance number, respectively.

In [25, 26] Shareshian and the second named author introduce a $q$ analog of the exc interpretation of $A_{n}(t)$ and in [29] they introduce a 
$q$-analog of the des interpretation of $A_{n}(t)$. These $q$-analogs are shown to be equal in [29, Theorem 9.7]. They are defined by

$$
A_{n}(q, t):=\sum_{\sigma \in \mathfrak{S}_{n}} q^{\operatorname{maj}(\sigma)-\operatorname{exc}(\sigma)} t^{\operatorname{exc}(\sigma)}=\sum_{\sigma \in \mathfrak{S}_{n}} q^{\operatorname{maj}_{\geq 2}\left(\sigma^{-1}\right)} t^{\operatorname{des}(\sigma)},
$$

where maj is MacMahon's classical major index and maj ${ }_{>2}$ is a permutation statistic whose definition is given in Section 6, By taking the stable principal specialization of both sides of (1.5), the following $q$-analog of Euler's classical formula is established in [26] for the exc interpretation of $A_{n}(q, t)$ and in [29] for the des interpretation:

$$
1+\sum_{n \geq 1} A_{n}(q, t) \frac{z^{n}}{[n]_{q} !}=\frac{(1-t) \exp _{q}(z)}{\exp _{q}(t z)-t \exp _{q}(z)}
$$

where

$$
[n]_{q} !:=[n]_{q}[n-1]_{q} \ldots[1]_{q} \quad \text { and } \quad \exp _{q}(z):=\sum_{n \geq 0} \frac{z^{n}}{[n]_{q} !} .
$$

In [24], Sagan, Shareshian and the second named author use the expansion (1.6) of $\omega W_{n}(\mathbf{x}, t)$ in the power sum basis to show that $A_{n}(q, t)$ evaluated at any $n$th root of unity is a polynomial in $t$ with positive integer coefficients. For results on cycle-type refinements of the exc interpretation of $A_{n}(q, t)$ see [29, 19, 24].

The Smirnov word enumerator $\tilde{W}_{n}^{\neq}(\mathbf{x}, t)$ arose in the work [9, 10, 11] of the first named author on chromatic quasisymmetric functions of directed graphs. The first named author proves the $t$-analog of (1.2),

$$
\sum_{n \geq 1} \tilde{W}_{n}^{\neq}(\mathbf{x}, t) z^{n}=\frac{\sum_{i \geq 2} i t[i-1]_{t} e_{i}(\mathbf{x}) z^{i}}{1-\sum_{i \geq 2} t[i-1]_{t} e_{i}(\mathbf{x}) z^{i}}
$$

from which e-positivity of $\tilde{W}_{n}^{\neq}(\mathbf{x}, t)$ follows. (A subsequent alternative proof of (1.9) was given in [1].) As a consequence of a general result obtained in [9, 10, 11] on power sum expansions of chromatic quasisymmetric functions, the first named author also obtains the following expansion analogous to (1.6):

$$
\omega \tilde{W}_{n}^{\neq}(\mathbf{x}, t)=\sum_{\substack{\lambda \vdash n \\ l(\lambda)>1}}\left(n t A_{l(\lambda)-1}(t) \prod_{i=1}^{l(\lambda)}\left[\lambda_{i}\right]_{t}\right) \frac{p_{\lambda}}{z_{\lambda}}+n t[n-1]_{t} \frac{p_{n}}{n} .
$$


1.2. New results. In this paper we obtain results for $\tilde{W}_{n}(\mathbf{x}, t)$ and $W_{n}^{\neq}(\mathbf{x}, t)$, analogous to those described above. For instance, we prove the $t$-analog of (1.1),

$$
\sum_{n \geq 1} \tilde{W}_{n}(\mathbf{x}, t) z^{n}=\frac{\sum_{i \geq 1} i t^{i-1} e_{i}(\mathbf{x}) z^{i}}{1-\sum_{i \geq 2} t[i-1]_{t} e_{i}(\mathbf{x}) z^{i}},
$$

and the $t$-analog of (1.2),

$$
\sum_{n \geq 1} W_{n}^{\neq}(\mathbf{x}, t) z^{n}=\frac{\sum_{i \geq 2}\left([i]_{t}+i t[i-2]_{t}\right) e_{i}(\mathbf{x}) z^{i}}{1-\sum_{i \geq 2} t[i-1]_{t} e_{i}(\mathbf{x}) z^{i}} .
$$

From this it follows that $\tilde{W}_{n}(\mathbf{x}, t)$ and $W_{n}^{\neq}(\mathbf{x}, t)$ are symmetric in $\mathbf{x}$ and $e$-positive. We also obtain expansions in the power sum symmetric functions analogous to (1.6) and (1.10).

Equation (1.11) can be restated as

$$
\sum_{n \geq 1} \tilde{W}_{n}(\mathbf{x}, t) z^{n}=\frac{(1-t) \frac{\partial}{\partial t} E(t z)}{E(t z)-t E(z)}
$$

By specializing (1.13), using an expansion of $\tilde{W}_{n}(\mathbf{x})$ in the fundamental quasisymmetric functions, we obtain the cyclic analog of (1.8),

$$
\sum_{n \geq 1}\left(\sum_{\sigma \in \mathfrak{S}_{n}} q^{\mathrm{maj}_{\geq 2}\left(\sigma^{-1}\right)} t^{\operatorname{cdes}(\sigma)}\right) \frac{z^{n}}{[n]_{q} !}=\frac{(1-t) \frac{\partial}{\partial t} \exp _{q}(t z)}{\exp _{q}(t z)-t \exp _{q}(z)}
$$

Our work relies on studying restricted Smirnov word enumerators that are components of all the Smirnov word enumerators discussed above. For $n \geq 1$, let

$$
\begin{aligned}
& W_{n}^{<}(\mathbf{x}, t):=\sum_{\substack{w \in W_{n} \\
w_{1}<w_{n}}} t^{\operatorname{des}(w)} x_{w} \\
& W_{n}^{>}(\mathbf{x}, t):=\sum_{\substack{w \in W_{n} \\
w_{1}>w_{n}}} t^{\operatorname{des}(w)} x_{w} \\
& W_{n}^{=}(\mathbf{x}, t):=\sum_{\substack{w \in W_{n} \\
w_{1}=w_{n}}} t^{\operatorname{des}(w)} x_{w} .
\end{aligned}
$$


Clearly

$$
\begin{aligned}
W_{n}(\mathbf{x}, t) & =W_{n}^{<}(\mathbf{x}, t)+W_{n}^{>}(\mathbf{x}, t)+W_{n}^{=}(\mathbf{x}, t) \\
\tilde{W}_{n}(\mathbf{x}, t) & =t W_{n}^{<}(\mathbf{x}, t)+W_{n}^{>}(\mathbf{x}, t)+W_{n}^{=}(\mathbf{x}, t) \\
W_{n}^{\neq}(\mathbf{x}, t) & =W_{n}^{<}(\mathbf{x}, t)+W_{n}^{>}(\mathbf{x}, t) \\
\tilde{W}_{n}^{\neq}(\mathbf{x}, t) & =t W_{n}^{<}(\mathbf{x}, t)+W_{n}^{>}(\mathbf{x}, t) .
\end{aligned}
$$

It is an exercise in [16, Exercise 2.9.11] that $W_{n}^{<}(\mathbf{x}, t), W_{n}^{>}(\mathbf{x}, t)$, and $W_{n}^{=}(\mathbf{x}, t)$ are symmetric in $\mathbf{x}$. Here we derive results for $W_{n}^{<}(\mathbf{x}, t)$, $W_{n}^{>}(\mathbf{x}, t)$, and $W_{n}^{=}(\mathbf{x}, t)$ analogous to those of $W_{n}(\mathbf{x}, t)$, which not only establish symmetry, but also $e$-positivity of $W_{n}^{<}(\mathbf{x}, t)$ and $W^{>}(\mathbf{x}, t)$. When appropriately combined they yield the above mentioned results for $\tilde{W}_{n}(\mathbf{x}, t)$ and $W_{n}^{\neq}(\mathbf{x}, t)$. They also enable us to recover the previous results for $W_{n}(\mathbf{x}, t)$ and $\tilde{W}_{n}^{\neq}(\mathbf{x}, t)$. However they do not provide new proofs of the previous results since their proofs rely on these results.

The Smirnov word enumerator is an example of a chromatic quasisymmetric function. (The chromatic quasisymmetric functions are a refinement of Stanley's chromatic symmetric functions, which were introduced by Shareshian and the second named author in [28, 29]). Indeed, $W_{n}(\mathbf{x}, t)$ is the chromatic quasisymmetric function of the naturally labeled path graph with $n$ nodes. In this paper our results for $W_{n}^{<}(\mathbf{x}, t)$ and $W_{n}^{>}(\mathbf{x}, t)$ are used to obtain new results in the study of chromatic quasisymmetric functions. For instance, we use $e$-positivity of $W_{n}^{<}(\mathbf{x}, t)$ and $W_{n}^{>}(\mathbf{x}, t)$ to establish $e$-positivity of the chromatic quasisymmetric function of the labeled cycle $C_{n}$, providing an example of an $e$-positive chromatic quasisymmetric function not covered by the refinement of the Stanley-Stembridge $e$-positivity conjecture appearing in [28, 29] nor by the directed graph version appearing in [9, 10, 11].

Smirnov words have been used in the literature to enumerate unconstrained words; see e.g. [12, 21, 22, 23]. In a forthcoming paper we use results discussed in this paper to obtain analogous results for unconstrained words.

\section{EXPANSION IN THE ELEMENTARY SYMMETRIC FUNCTIONS}

In this section we derive formulas that refine (1.4) and (1.9) and then use the refinements to prove (1.11) and (1.12). 
Theorem 2.1. Let

$$
\begin{aligned}
D(\mathbf{x}, t, z) & :=1-\sum_{i \geq 2} t[i-1]_{t} e_{i}(\mathbf{x}) z^{i}, \\
a_{i}(t) & :=\frac{d}{d t}[i]_{t}=\sum_{j=0}^{i-2}(j+1) t^{j}, \\
b_{i}(t) & :=t^{i-1} a_{i}\left(t^{-1}\right)=\sum_{j=1}^{i-1}(i-j) t^{j}, \\
c_{i}(t) & :=i t[i-2]_{t},
\end{aligned}
$$

for all $i \geq 2$. Then

$$
\begin{aligned}
\sum_{n \geq 1} W_{n}^{<}(\mathbf{x}, t) z^{n} & =\frac{1}{D(\mathbf{x}, t, z)} \sum_{i \geq 2} a_{i}(t) e_{i}(\mathbf{x}) z^{i} \\
\sum_{n \geq 1} W_{n}^{>}(\mathbf{x}, t) z^{n} & =\frac{1}{D(\mathbf{x}, t, z)} \sum_{i \geq 2} b_{i}(t) e_{i}(\mathbf{x}) z^{i} \\
\sum_{n \geq 1} W_{n}^{=}(\mathbf{x}, t) z^{n} & =\frac{1}{D(\mathbf{x}, t, z)}\left(e_{1}(\mathbf{x}) z-\sum_{i \geq 2} c_{i}(t) e_{i}(\mathbf{x}) z^{i}\right) .
\end{aligned}
$$

Before proving the theorem, we observe that

$$
\begin{aligned}
a_{i}(t)+b_{i}(t) & =1+(i+1) t+(i+1) t^{2}+\cdots+(i+1) t^{i-2}+t^{i-1} \\
& =[i]_{t}+i t[i-2]_{t} .
\end{aligned}
$$

Hence,

$$
a_{i}(t)+b_{i}(t)-c_{i}(t)=[i]_{t},
$$

which shows that Theorem 2.1 refines (1.4) since $W_{n}^{<}(\mathbf{x}, t)+W_{n}^{>}(\mathbf{x}, t)+$ $W_{n}^{=}(\mathbf{x}, t)=W_{n}(\mathbf{x}, t)$. Also

$$
t a_{i}(t)+b_{i}(t)=i t[i-1]_{t},
$$

which shows that Theorem 2.1 also refines (1.9) since $t W_{n}^{<}(\mathbf{x}, t)+$ $W_{n}^{>}(\mathbf{x}, t)=\tilde{W}_{n}^{\neq}(\mathbf{x}, t)$.

We prove (2.4) first. Then we use (2.4), (1.4), and (1.9) to derive (2.2). Equation (2.3) follows from (2.2). Our proof of (2.4) uses the transfer-matrix method discussed in [33, Section 4.7] and borrows ingredients from the first named author's proof of (1.9) in [10].

Before presenting the proof of (2.4), we give a brief review of the transfer matrix method. A walk of length $n$ on a directed graph $G=$ $([k], E)$ is a sequence of vertices $v_{0}, v_{1}, \ldots, v_{n}$ such that $\left(v_{i-1}, v_{i}\right) \in E$ for all $i \in[n]$. A walk is closed if $v_{0}=v_{n}$. We attach weights in some commutative ring $R$ to the edges of $G$. Let wt $: E \rightarrow R$ be the weight 
function. Now define the weight $\operatorname{wt}(w)$ of a walk $w:=v_{0}, v_{1}, \ldots, v_{n}$ to be the product $\operatorname{wt}\left(v_{0}, v_{1}\right) \mathrm{wt}\left(v_{1}, v_{2}\right) \ldots \operatorname{wt}\left(v_{n-1}, v_{n}\right)$. For each $i, j \in[k]$, define $\mathcal{W}_{i, j, n}$ to be the set of walks of length $n$ from $i$ to $j$ and let

$$
U_{i, j, n}:=\sum_{w \in \mathcal{W}_{i, j, n}} \operatorname{wt}(w)
$$

The transfer-matrix method enables one to express the generating function $\sum_{n \geq 0} U_{i, j, n} z^{n}$ in terms of the adjacency matrix $A$ for the edge weighted digraph $G$. That is, $A$ is the $k \times k$ matrix whose $(i, j)$-entry is

$$
A_{i, j}:= \begin{cases}\mathrm{wt}(i, j) & \text { if }(i, j) \in E \\ 0 & \text { otherwise. }\end{cases}
$$

Theorem 4.7.2 of [33] states that for all $i, j \in[k]$,

$$
\sum_{n \geq 0} U_{i, j, n} z^{n}=\frac{(-1)^{i+j} \operatorname{det}(I-z A: j, i)}{\operatorname{det}(I-z A)}
$$

where $(B: i, j)$ is the matrix obtained from $B$ by removing row $i$ and column $j$.

Proof of 2.4). As in [32] and [10], we view a Smirnov word $w_{1} w_{2} \ldots w_{n}$ over the alphabet $[k]$ as a walk $w_{1}, w_{2}, \ldots, w_{n}$ of length $n-1$ on the digraph $G=([k], E)$, where

$$
E=\{(i, j): i, j \in[k] \text { and } i \neq j\}
$$

We let the edge weights belong to the commutative ring $\mathbb{Z}\left[x_{1}, \ldots, x_{k}, t\right]$ and for all $(i, j) \in E$, and we set

$$
\mathrm{wt}(i, j):= \begin{cases}x_{j} & \text { if } i<j \\ t x_{j} & \text { if } i>j\end{cases}
$$

Note that if $w$ is a Smirnov word over the alphabet $[k]$ then

$$
t^{\operatorname{des}(w)} x_{w}=x_{w_{1}} \operatorname{wt}(w),
$$

where $w_{1}$ is the first letter of $w$. Hence

$$
W_{n}^{=}\left(x_{1}, \ldots, x_{k}, t\right):=W_{n}^{=}\left(x_{1}, \ldots, x_{k}, 0,0, \ldots, t\right)=\sum_{i=1}^{k} x_{i} U_{i, i, n-1} .
$$


It follows from this and (2.5) that

$$
\begin{aligned}
\sum_{n \geq 1} W_{n}^{=}\left(x_{1}, \ldots, x_{k}, t\right) z^{n} & =z \sum_{i=1}^{k} x_{i} \sum_{n \geq 0} U_{i, i, n} z^{n} \\
& =z \sum_{i=1}^{k} x_{i} \frac{\operatorname{det}(I-z A: i, i)}{\operatorname{det}(I-z A)} \\
& =\frac{z \sum_{i=1}^{k} x_{i} \operatorname{det}(I-z A: i, i)}{\operatorname{det}(I-z A)}
\end{aligned}
$$

where

$$
A=\left[\begin{array}{ccccc}
0 & x_{2} & x_{3} & \ldots & x_{k} \\
t x_{1} & 0 & x_{3} & \ldots & x_{k} \\
t x_{1} & t x_{2} & 0 & \ldots & x_{k} \\
\vdots & \vdots & \vdots & \ddots & \vdots \\
t x_{1} & t x_{2} & t x_{3} & \ldots & 0
\end{array}\right]
$$

In [10, Proof of Theorem 6.1] the first named author proves that 1

$$
\operatorname{det}(I-z A)=1-\sum_{j \geq 2} e_{j}\left(x_{1}, \ldots, x_{k}\right) t[j-1]_{t} z^{j}
$$

It follows that

$$
\operatorname{det}(I-z A: i, i)=1-\sum_{j \geq 2} e_{j}\left(x_{1}, \ldots, \hat{x}_{i}, \ldots, x_{k}\right) t[j-1]_{t} z^{j},
$$

where $\hat{x}_{i}$ denotes deletion of $x_{i}$. Multiplying both sides by $x_{i}$ and summing over all $i \in[k]$ yields,

$$
\sum_{i=1}^{k} x_{i} \operatorname{det}(I-z A: i, i)=\sum_{i=1}^{k} x_{i}-\sum_{j \geq 2} \sum_{i=1}^{k} x_{i} e_{j}\left(x_{1}, \ldots, \hat{x}_{i}, \ldots, x_{k}\right) t[j-1]_{t} z^{j} .
$$

One can see that

$$
\sum_{i=1}^{k} x_{i} e_{j}\left(x_{1}, \ldots, \hat{x}_{i}, \ldots, x_{k}\right)=(j+1) e_{j+1}\left(x_{1}, \ldots, x_{k}\right)
$$

since both sides enumerate $(j+1)$-subsets of $[k]$ with a distinguished element. Hence

$\sum_{i=1}^{k} x_{i} \operatorname{det}(I-z A: i, i)=e_{1}\left(x_{1}, \ldots, x_{k}\right)-\sum_{j \geq 2}(j+1) e_{j+1}\left(x_{1}, \ldots, x_{k}\right) t[j-1]_{t} z^{j}$.

\footnotetext{
${ }^{1}$ This is obtained from the formula in [10] by replacing $t$ with $t^{-1}$ and each $x_{i}$ with $t x_{i}$
} 
Upon multiplying both sides by $z$, we see that the numerator of the right hand side of (2.6) is

$$
e_{1}\left(x_{1}, \ldots, x_{k}\right) z-\sum_{j \geq 3} j e_{j}\left(x_{1}, \ldots, x_{k}\right) t[j-2]_{t} z^{j} .
$$

It therefore follows from (2.6) and (2.7) that

$$
\sum_{n \geq 1} W_{n}^{=}\left(x_{1}, \ldots, x_{k}, t\right) z^{n}=\frac{e_{1}\left(x_{1}, \ldots, x_{k}\right) z-\sum_{j \geq 3} j e_{j}\left(x_{1}, \ldots, x_{k}\right) t[j-2]_{t} z^{j}}{1-\sum_{j \geq 2} e_{j}\left(x_{1}, \ldots, x_{k}\right) t[j-1]_{t} z^{j}} .
$$

The desired result (2.4) follows by taking the limit as $k$ goes to infinity.

Proof of (2.2). It follows from (1.15), (1.4), and (2.4) that

$$
\begin{aligned}
\sum_{n \geq 1} W_{n}^{<}(\mathbf{x}, t) z^{n}+\sum_{n \geq 1} W_{n}^{>}(\mathbf{x}, t) z^{n} & =\sum_{n \geq 1} W_{n}(\mathbf{x}, t)-\sum_{n \geq 1} W_{n}^{=}(\mathbf{x}, t) \\
& =\frac{B(\mathbf{x}, t, z)}{D(\mathbf{x}, t, z)}
\end{aligned}
$$

where

$$
\begin{aligned}
B(\mathbf{x}, t, z) & =\sum_{i \geq 1}[i]_{t} e_{i}(\mathbf{x}) z^{i}-\left(e_{1}(\mathbf{x}) z-\sum_{i \geq 2} i t[i-2]_{t} e_{i}(\mathbf{x}) z^{i}\right) \\
& =\sum_{i \geq 2}[i]_{t} e_{i}(\mathbf{x}) z^{i}+\sum_{i \geq 2} i t[i-2]_{t} e_{i}(\mathbf{x}) z^{i} \\
& =\sum_{i \geq 2}\left([i]_{t}+i t[i-2]_{t}\right) e_{i}(\mathbf{x}) z^{i} .
\end{aligned}
$$

It follows from (1.16) and (1.9) that

$$
\begin{aligned}
t \sum_{n \geq 1} W_{n}^{<}(\mathbf{x}, t) z^{n}+\sum_{n \geq 1} W_{n}^{>}(\mathbf{x}, t) z^{n} & =\sum_{n \geq 1} \tilde{W}_{n}^{\neq}(\mathbf{x}, t) z^{n} \\
& =\frac{C(\mathbf{x}, t, z)}{D(\mathbf{x}, t, z)}
\end{aligned}
$$

where

$$
C(\mathbf{x}, t, z)=\sum_{i \geq 2} i t[i-1]_{t} e_{i}(\mathbf{x}) z^{i}
$$


By subtracting (2.8) from (2.9), we obtain

$$
\begin{aligned}
(t-1) \sum_{n \geq 1} W_{n}^{<}(\mathbf{x}, t) z^{n} & =\frac{C(\mathbf{x}, t, z)-B(\mathbf{x}, t, z)}{D(\mathbf{x}, t, z)} \\
& =\frac{\sum_{i \geq 2}\left(i t^{i-1}-[i]_{t}\right) e_{i}(\mathbf{x}) z^{i}}{D(\mathbf{x}, t, z)} .
\end{aligned}
$$

Note that

$$
\begin{aligned}
(t-1)\left(1+2 t+3 t^{2}+\cdots+(i-1) t^{i-2}\right) & =(i-1) t^{i-1}-[i-1]_{t} \\
& =i t^{i-1}-[i]_{t} .
\end{aligned}
$$

Hence

$$
\sum_{n \geq 1} W_{n}^{<}(\mathbf{x}, t) z^{n}=\frac{\sum_{i \geq 2}\left(1+2 t+3 t^{2}+\cdots+(i-1) t^{i-2}\right) e_{i}(\mathbf{x}) z^{i}}{D(\mathbf{x}, t, z)}
$$

as desired.

Proof of (2.3). Using the involution on the set of Smirnov words that reverses each word, we see that $W_{n}^{>}(\mathbf{x}, t)=t^{n-1} W_{n}^{<}\left(\mathbf{x}, t^{-1}\right)$. It follows from this and (2.2) that

$$
\begin{aligned}
\sum_{n \geq 1} W_{n}^{>}(\mathbf{x}, t) z^{n} & =t^{-1} \sum_{n \geq 1} W_{n}^{<}\left(\mathbf{x}, t^{-1}\right)(t z)^{n} \\
& =t^{-1} \frac{\sum_{i \geq 2} a_{i}\left(t^{-1}\right) e_{i}(\mathbf{x}) t^{i} z^{i}}{D\left(\mathbf{x}, t^{-1}, t z\right)} \\
& =\frac{\sum_{i \geq 2} b_{i}(t) e_{i}(\mathbf{x}) z^{i}}{D\left(\mathbf{x}, t^{-1}, t z\right)} .
\end{aligned}
$$

Since $D\left(\mathbf{x}, t^{-1}, t z\right)=D(\mathbf{x}, t, z)$, the result holds.

Since

$$
\frac{1}{D(\mathbf{x}, t, z)}=\sum_{m \geq 0}\left(\sum_{i \geq 2} t[i-1]_{t} e_{i}(\mathbf{x}) z^{i}\right)^{m},
$$

we have the following consequence of Theorem 2.1.

Corollary 2.2. For all $n \geq 1$, the polynomials $W_{n}^{<}(\mathbf{x}, t)$ and $W_{n}^{>}(\mathbf{x}, t)$ are e-positive.

Note that it follows from Theorem 2.1 that the coefficient of $e_{n}(\mathbf{x})$ in the $e$-basis expansion of $W_{n}^{=}(\mathbf{x}, t)$ is $-n t[n-2]_{t}$ if $n \geq 2$. Hence $W_{n}^{=}(\mathbf{x}, t)$ fails to be $e$-positive. However, observe that the coefficient $c_{\lambda}(t)$ of $e_{\lambda}(\mathbf{x})$ is in $\mathbb{N}[t]$ if the smallest part of $\lambda$ is 1 , and $-c_{\lambda}(t) \in \mathbb{N}[t]$ otherwise. 
We obtain equivalent formulations of (2.2) and of (2.3) by multiplying the numerators and denominators of the right hand sides of the equations by $1-t$.

Corollary 2.3. We have

$$
\begin{aligned}
& \sum_{n \geq 1} W_{n}^{<}(\mathbf{x}, t) z^{n}=\frac{(1-t) \frac{\partial}{\partial t} \sum_{i \geq 2}[i]_{t} e_{i}(\mathbf{x}) z^{i}}{E(t z)-t E(z)} \\
& \sum_{n \geq 1} W_{n}^{>}(\mathbf{x}, t) z^{n}=\frac{(1-t) \sum_{i \geq 2}\left(\sum_{j=1}^{i-1}(i-j) t^{j}\right) e_{i}(\mathbf{x}) z^{i}}{E(t z)-t E(z)}
\end{aligned}
$$

where $E(z):=\sum_{n \geq 0} e_{n}(\mathbf{x}) z^{n}$.

We now use Theorem 2.1 to prove (1.12) and (1.11), which we restate here.

Corollary 2.4. We have,

$$
\sum_{n \geq 1} W_{n}^{\neq}(\mathbf{x}, t) z^{n}=\frac{\sum_{i \geq 2}\left([i]_{t}+i t[i-2]_{t}\right) e_{i}(\mathbf{x}) z^{i}}{D(\mathbf{x}, t, z)} .
$$

Consequently, $W_{n}^{\neq}(\mathbf{x}, t)$ is e-positive.

Proof. We use the facts that $W_{n}^{\neq}(\mathbf{x}, t)=W_{n}^{<}(\mathbf{x}, t)+W_{n}^{>}(\mathbf{x}, t)$ and $a_{i}(t)+b_{i}(t)=[i]_{t}+i t[i-2]_{t}$.

Corollary 2.5. We have,

$$
\sum_{n \geq 1} \tilde{W}_{n}(\mathbf{x}, t) z^{n}=\frac{\sum_{i \geq 1} i t^{i-1} e_{i}(\mathbf{x}) z^{i}}{D(\mathbf{x}, t, z)} .
$$

Consequently, $\tilde{W}_{n}(\mathbf{x}, t)$ is e-positive.

Proof. We use the fact that

$$
\tilde{W}_{n}(\mathbf{x}, t)=t W_{n}^{<}(\mathbf{x}, t)+\left(W_{n}(\mathbf{x}, t)-W_{n}^{<}(\mathbf{x}, t)\right)
$$

and equations (2.2), (2.10), and (1.4).

Corollary 2.6. We have,

$$
\sum_{n \geq 1} \tilde{W}_{n}(\mathbf{x}, t) z^{n}=\frac{(1-t) \frac{\partial}{\partial t} E(t z)}{E(t z)-t E(z)} .
$$

In [26, Theorem 5.1] (see also [29, Corollary C.5]), it is observed that (1.4) implies that the Smirnov enumerator $W_{n}(\mathbf{x}, t)$ has a stronger property than $e$-positivity, namely $e$-unimodality, and in [9, 10] the same is observed for $\tilde{W}_{n}^{\neq}(\mathbf{x}, t)$ as a consequence of (1.9). Here we show that $W_{n}^{\neq}(\mathbf{x}, t)$ also has the stronger property, while $\tilde{W}_{n}(\mathbf{x}, t)$ does not. 
A polynomial $A(t)=a_{0}+a_{1} t+\cdots+a_{n} t^{n} \in \mathbb{Q}[t]$ is said to be unimodal if

$$
0 \leq a_{0} \leq a_{1} \leq \cdots \leq a_{c} \geq a_{c+1} \geq a_{c+2} \geq \cdots \geq a_{n} \geq 0,
$$

for some $c$. Let $\Lambda_{\mathbb{Q}}$ denote the $\mathbb{Q}$-algebra of symmetric functions over $\mathbf{x}:=x_{1}, x_{2}, \ldots$ Given $r, s \in \Lambda_{\mathbb{Q}}$, we say that $r \leq_{e} s$ if $s-r$ is $e$ positive. A polynomial $A(t)=a_{0}+a_{1} t+\cdots+a_{n} t^{n} \in \Lambda_{\mathbb{Q}}[t]$ is said to be e-unimodal if

$$
0 \leq_{e} a_{0} \leq_{e} a_{1} \leq_{e} \cdots \leq_{e} a_{c} \geq_{e} a_{c+1} \geq_{e} a_{c+2} \geq_{e} \cdots \geq_{e} a_{n} \geq_{e} 0,
$$

for some $c$. We say that $A(t)$ (over any coefficient ring) is palindromic with center of symmetry $\frac{n}{2}$ if $a_{j}=a_{n-j}$ for $0 \leq j \leq n$. (Note that the center of symmetry is unique unless $A(t)$ is the zero polynomial, in which case every number of the form $\frac{n}{2}$, where $n \in \mathbb{N}$, satisfies the definition of center of symmetry.) It is easy to see that $A(t) \in$ $\Lambda_{\mathbb{Q}}[t]$ is $e$-unimodal and palindromic with center of symmetry $c$ if and only if all the coefficients in the $e$-expansion of $A(t)$ are unimodal and palindromic polynomials in $t$ with the same center of symmetry $c$; see [29, Proposition B.3].

Lemma 2.7. Let $\left(g_{n}(t)\right)_{n \geq 2}$ be a sequence of polynomials in $\mathbb{Q}[t]$, such that each $g_{n}(t)$ is unimodal and palindromic with center of symmetry $\frac{n+r}{2}$, where $r \geq-2$ is a fixed integer. If $\left(G_{n}(\mathbf{x}, t)\right)_{n \geq 2}$ is a sequence of polynomials in $\Lambda_{\mathbb{Q}}[t]$ that satisfies

$$
\sum_{n \geq 2} G_{n}(\mathbf{x}, t) z^{n}=\frac{\sum_{n \geq 2} g_{n}(t) e_{n}(\mathbf{x}) z^{n}}{D(\mathbf{x}, t, z)}
$$

where $D(\mathbf{x}, t, z)$ is defined in (2.1), then each $G_{n}(\mathbf{x}, t)$ is e-unimodal and palindromic with center of symmetry $\frac{n+r}{2}$.

Proof. We use Propositions B.1 and B.3 of [29]. By (2.11),

$$
G_{n}(\mathbf{x}, t)=\sum_{m \geq 1} \sum_{\substack{k_{1}, \ldots, k_{m} \geq 2 \\ \sum_{i=1}^{m} k_{i}=n}} e_{k_{1}} \ldots e_{k_{m}} t^{m-1} g_{k_{1}}(t) \prod_{i=2}^{m}\left[k_{i}-1\right]_{t} .
$$

For each nonzero $g_{k_{1}}(t)$, the polynomial $t^{m-1} g_{k_{1}}(t) \prod_{i=2}^{m}\left[k_{i}-1\right]_{t}$ is a product of palindromic, unimodal polynomials. Hence, the product is also palindromic and unimodal with center of symmetry equal to

$$
m-1+\frac{k_{1}+r}{2}+\sum_{i=2}^{m} \frac{k_{i}-2}{2}=\frac{n+r}{2} .
$$

\footnotetext{
${ }^{2}$ Note that our definition is nonstandard in that positivity of the coefficients is also required.
} 
Since each such product has the same center of symmetry, $G_{n}(\mathbf{x}, t)$ is palindromic and $e$-unimodal with center of symmetry $\frac{n+r}{2}$.

Corollary 2.8 (of Corollary 2.4). For all $n \geq 2$, the Smirnov word enumerator $W_{n}^{\neq}(\mathbf{x}, t)$ is e-unimodal and palindromic with center of symmetry $\frac{n-1}{2}$.

Proof. Since $[i]_{t}+i t[i-2]_{t}$ is unimodal and palindromic with center of symmetry $\frac{i-1}{2}$, the result follows from Lemma 2.7

We note that although $W_{n}(\mathbf{x}, t), W_{n}^{\neq}(\mathbf{x}, t)$, and $\tilde{W}^{\neq}(\mathbf{x}, t)$ are all $e$ unimodal and palindromic, this is not the case for $\tilde{W}_{n}(\mathbf{x}, t)$. Indeed, it follows from Corollary 2.5 that

$$
\tilde{W}_{5}(\mathbf{x}, t)=e_{4,1} t+\left(e_{2,2,1}+e_{4,1}+2 e_{3,2}\right) t^{2}+\left(e_{4,1}+5 e_{3,2}\right) t^{3}+\left(5 e_{5}\right) t^{4},
$$

which is neither palindromic nor $e$-unimodal. However, for certain partitions $\lambda$, the coefficient of $e_{\lambda}$ is a palindromic and unimodal polynomial in $t$.

Corollary 2.9 (of Corollary 2.5). For $\lambda \vdash n$, let $c_{\lambda}(t)$ be the coefficient of $e_{\lambda}$ in the expansion of $\tilde{W}_{n}(\mathbf{x}, t)$ in the elementary symmetric functions. If $\lambda=\left(\lambda_{1} \geq \cdots \geq \lambda_{k-1} \geq 1\right)$ then

$$
c_{\lambda}(t)=t^{k-1} \prod_{i=1}^{k-1}\left[\lambda_{i}-1\right]_{t}
$$

which is palindromic and unimodal. If $\lambda=j^{k}$ then

$$
c_{\lambda}(t)=j t^{j+k-2}[j-1]_{t}^{k-1},
$$

which is palindromic and unimodal.

\section{Chromatic quasisymmetric function of the CyCle}

In this section, we discuss the connection between the Smirnov word enumerators and the chromatic quasisymmetric functions introduced by Shareshian and the second named author in [28, 29]. We use results of the previous section to provide an example of an e-positive chromatic quasisymmetric function not covered by the refinement of the StanleyStembridge $e$-positivity conjecture appearing in [28, 29] or its directed graph extension appearing in [9, 10]. 
For any graph $G=([n], E)$, define the chromatic quasisymmetric function ${ }^{3}$ of $G$ as

$$
X_{G}(\mathbf{x}, t):=\sum_{\kappa \in \mathcal{C}(G)} t^{\operatorname{des}(\kappa)} x_{\kappa(1)} x_{\kappa(2)} \cdots x_{\kappa(n)},
$$

where $\mathcal{C}(G)$ is the set of proper colorings $\kappa:[n] \rightarrow \mathbb{P}$ of $G$ and

$$
\operatorname{des}(\kappa):=\mid\{\{i, j\} \in E: i<j \text { and } \kappa(i)>\kappa(j)\} \mid .
$$

When $t=1, X_{G}(\mathbf{x}, t)$ is Stanley's chromatic symmetric function $X_{G}(\mathbf{x})$. Note that $X_{G}(\mathbf{x}, t)$ is a polynomial in $t$ whose coefficients are quasisymmetric functions. We view $G$ as a labeled graph and note that the definition of $X_{G}(\mathbf{x}, t)$ depends on the vertex labeling, and not just on the isomorphism class of $G$, as is the case for $X_{G}(\mathbf{x})$.

Since Smirnov words of length $n$ can be viewed as proper colorings of the naturally labeled path

$$
P_{n}:=([n],\{\{i, i+1\}: i \in[n-1]\}),
$$

it follows that

$$
W_{n}(\mathbf{x}, t)=X_{P_{n}}(\mathbf{x}, t) .
$$

The Smirnov word enumerator $\tilde{W}_{n}^{\neq}(\mathbf{x}, t)$ can also be viewed as a chromatic quasisymmetric function, but in the more general sense considered by the first named author [9, 10], in which labeled graphs are replaced by directed graphs and the definition of $\operatorname{des}(\kappa)$ given in (3.1) is replaced by

$$
\operatorname{des}(\kappa):=|\{(i, j) \in E: \kappa(i)>\kappa(j)\}| .
$$

Labeled graphs can be viewed as directed graphs by orienting each edge from smaller vertex to larger vertex; so the digraph version of chromatic quasisymmetric function is more general than the labeled graph version. One can see that

$$
\tilde{W}_{n}^{\neq}(\mathbf{x}, t)=X_{\vec{C}_{n}}(\mathbf{x}, t),
$$

where $\vec{C}_{n}$ is the directed cycle defined by

$$
\vec{C}_{n}:=([n],\{(i, i+1): i \in[n-1]\} \cup\{(n, 1)\}) .
$$

The longstanding Stanley-Stembridge conjecture [32 asserts that $X_{G}(\mathbf{x})$ is $e$-positive when $G$ is the incomparability graph of a $(3+1)$ free poset. In [17], Guay-Paquet proves that if the Stanley-Stembridge conjecture holds for incomparability graphs of posets that are both

\footnotetext{
${ }^{3}$ In the definition given in [29] ascents are counted instead of descents, but in the case that $X_{G}(\mathbf{x}, t)$ is symmetric, it is shown in [29, Corollary 2.7] that the definitions are equivalent.
} 
$(3+1)$-free and $(2+2)$-free (known as unit interval graphs) then it holds in general.

In 29] Shareshian and the second named author show that $X_{G}(\mathbf{x}, t)$ is symmetric when $G$ is a unit interval graph with a certain natural labeling; these are called natural unit interval graphs. They also conjecture that $X_{G}(\mathbf{x}, t)$ is $e$-positive and $e$-unimodal when $G$ is a natural unit interval graph. The path $P_{n}$ is an example of a natural unit interval graph for which the conjecture holds since $X_{P_{n}}(\mathbf{x}, t)=W_{n}(\mathbf{x}, t)$. The symmetry result and $e$-positivity conjecture of [29] are generalized in [9, 10] to a class of directed graphs called indifference digraphs in [10. With the view that a labeled graph is an acyclic digraph, the natural unit interval graphs form the class of acyclic indifference digraphs. The directed cycle $\vec{C}_{n}$ is an example of an indifference digraph for which the extended conjecture holds since $X_{\vec{C}_{n}}(\mathbf{x}, t)=\tilde{W}_{n}^{\neq}(\mathbf{x}, t)$.

Here we consider the labeled cycle

$$
C_{n}:=([n],\{\{i, i+1\}: i \in[n-1]\} \cup\{\{1, n\}\}) .
$$

If we view $C_{n}$ as a directed graph (by orienting its edges from smaller vertex to larger vertex), we get a directed graph that is identical to the directed cycle $\vec{C}_{n}$ except for the edge $(n, 1)$ in $\vec{C}_{n}$ which is oriented as $(1, n)$ in $C_{n}$. For $n \geq 4$, the labeled cycle $C_{n}$ is not a natural unit interval graph, nor is it an indifference digraph. Nevertheless, since

$$
X_{C_{n}}(\mathbf{x}, t)=W_{n}^{<}(\mathbf{x}, t)+t W_{n}^{>}(\mathbf{x}, t),
$$

it follows from Corollary 2.2 that $X_{C_{n}}(\mathbf{x}, t)$ is symmetric and $e$-positive. This shows that the class of labeled graphs with $e$-positive chromatic quasisymmetric function is strictly larger than the class of natural unit interval graphs, and the class of digraphs with $e$-positive chromatic quasisymmetric function is strictly larger than the class of indifference digraphs.

Next we address the question of $e$-unimodality of $X_{C_{n}}(\mathbf{x}, t)$. From Theorem 2.1 and equation (3.3), we obtain the next result. For $n>0$, let

$$
[-n]_{t}:=\frac{t^{-n}-1}{t-1}=-t^{-n}[n]_{t} .
$$

Corollary 3.1 (of Theorem 2.11). We have,

$$
\sum_{n \geq 2} X_{C_{n}}(\mathbf{x}, t) z^{n}=\frac{\sum_{i \geq 2}\left([2]_{t}[i]_{t}+i t^{2}[i-3]_{t}\right) e_{i}(\mathbf{x}) z^{i}}{D(\mathbf{x}, t, z)}
$$

where $D(\mathbf{x}, t, z)$ is defined in (2.1).

Theorem 3.2. Let $n \geq 2$. 
(1) If $n$ is odd, $X_{C_{n}}(\mathbf{x}, t)$ is e-unimodal and palindromic with center of symmetry $\frac{n}{2}$.

(2) If $n$ is even,

(a) $X_{C_{n}}(\mathbf{x}, t)$ is e-positive and palindromic with center of symmetry $\frac{n}{2}$, but is not e-unimodal.

(b) $X_{C_{n}}(\mathbf{x}, t)+t^{\frac{n}{2}} e_{2^{\frac{n}{2}}}(\mathbf{x})$ is e-unimodal and palindromic with center of symmetry $\frac{n}{2}$.

Proof. Let $U_{n}(\mathbf{x}, t)$ and $V_{n}(\mathbf{x}, t)$ be defined respectively by

$$
\sum_{n \geq 2} U_{n}(\mathbf{x}, t) z^{n}=\frac{\left([2]_{t}[2]_{t}+2 t^{2}[2-3]_{t}\right) e_{2}(\mathbf{x}) z^{2}}{D(\mathbf{x}, t, z)}
$$

and

$$
\sum_{n \geq 2} V_{n}(\mathbf{x}, t) z^{n}=\frac{\sum_{i \geq 3}\left([2]_{t}[i]_{t}+i t^{2}[i-3]_{t}\right) e_{i}(\mathbf{x}) z^{i}}{D(\mathbf{x}, t, z)} .
$$

Then $X_{C_{n}}(\mathbf{x}, t)=U_{n}(\mathbf{x}, t)+V_{n}(\mathbf{x}, t)$.

We have,

$$
\sum_{n \geq 2} U_{n}(\mathbf{x}, t) z^{n}=\frac{\left(1+t^{2}\right) e_{2}(\mathbf{x}) z^{2}}{D(\mathbf{x}, t, z)} .
$$

It follows from (2.11) that

$$
U_{n}(\mathbf{x}, t)=\sum_{m \geq 1} \sum_{\substack{k_{2}, \ldots, k_{m} \geq 2 \\ \sum_{i=2}^{m} k_{i}=n-2}} e_{2} e_{k_{2}} \ldots e_{k_{m}} t^{m-1}\left(1+t^{2}\right) \prod_{i=2}^{m}\left[k_{i}-1\right]_{t}
$$

Note that for any $k \geq 3$

$$
\left(1+t^{2}\right)[k]_{t}=1+t+2 t^{2}+\cdots+2 t^{k-1}+t^{k}+t^{k+1}
$$

and for $k=2$,

$$
\left(1+t^{2}\right)[k]_{t}=1+t+t^{2}+t^{3} .
$$

In either case, $\left(1+t^{2}\right)[k]_{t}$ is unimodal and palindromic with center of symmetry $\frac{k+1}{2}$.

We now use Propositions B.1 and B.3 of [29]. Consider the term of the right side of (3.5) corresponding to the $(m-1)$-tuple $\left(k_{2}, \ldots, k_{m}\right)$. If $k_{j} \geq 3$ for some $j \geq 2$ then since $\left(1+t^{2}\right)\left[k_{j}-1\right]_{t}$ is unimodal and palindromic, $t^{m-1}\left(1+t^{2}\right) \prod_{i=2}^{m}\left[k_{i}-1\right]_{t}$ is a product of unimodal and palindromic polynomials. Hence $t^{m-1}\left(1+t^{2}\right) \prod_{i=2}^{m}\left[k_{i}-1\right]_{t}$ is unimodal and palindromic with center of symmetry

$$
m-1+\frac{k_{j}}{2}+\sum_{\substack{i=2 \\ i \neq j}}^{m} \frac{k_{i}-2}{2}=\frac{n}{2}
$$


It follows that if $\lambda$ has a part of size at least 3 then the coefficient of $e_{\lambda}$ in $U_{n}(\mathbf{x}, t)$ is palindromic and unimodal with center of symmetry $\frac{n}{2}$. If $\lambda$ does not have a part of size at least 3 then all the parts must be 2 , which means that $n$ is even. Hence if $n$ is odd then $U_{n}(\mathbf{x}, t)$ is $e$-unimodal and palindromic with center of symmetry $\frac{n}{2}$.

Now if $\lambda$ does not have a part of size at least 3 then $\lambda=2^{m}$, where $n=2 m$. By (3.5), the coefficient of $e_{\lambda}$ in $U_{n}(\mathbf{x}, t)$ is $t^{m-1}\left(1+t^{2}\right)$. Hence if $\lambda$ does not have a part of size at least 3 then coefficient of $e_{\lambda}$ in $U_{n}(\mathbf{x}, t)+t^{m} e_{2^{m}}$ is unimodal and palindromic with center of symmetry $m=\frac{n}{2}$. From the argument in the previous paragraph, the same is true if $\lambda$ has a part of size at least 3. It follows that if $n$ is even, $U_{n}(\mathbf{x}, t)+t^{m} e_{2^{m}}$ is $e$-unimodal and palindromic with center of symmetry $m=\frac{n}{2}$.

It follows from Lemma 2.7 that $V_{n}(\mathbf{x}, t)$ is also palindromic and $e$ unimodal with center of symmetry $\frac{n}{2}$. Since $X_{C_{n}}(\mathbf{x}, t)=U_{n}(\mathbf{x}, t)+$ $V_{n}(\mathbf{x}, t)$, Parts (1) and (2b) hold. Palindromicity of $X_{C_{n}}(\mathbf{x}, t)$ in the even case follows from Part (2b). The assertion in Part (2a) that $X_{C_{n}}(\mathbf{x}, t)$ is not $e$-unimodal in the even case follows from the fact the coefficient of $e_{2^{m}}(\mathbf{x})$ is $t^{m-1}\left(1+t^{2}\right)$, which is not unimodal.

\section{EXPANSION IN THE POWER SUM SYMMETRIC FUNCTIONS}

Let $A_{n}(t)$ be the Eulerian polynomial defined in (1.7) for $n \geq 1$ and let

$$
A_{0}(t):=t^{-1}
$$

Also let $\omega$ be the standard involution on $\Lambda_{\mathbb{Q}}$ taking the elementary symmetric function $e_{n}$ to the complete homogeneous symmetric function $h_{n}$, and let

$$
H(z):=\sum_{n \geq 0} h_{n}(\mathbf{x}) z^{n}
$$

where $h_{n}(\mathbf{x})$ is the complete homogenous symmetric function of degree n. Stembridge [35, Proposition 3.3] proves that

$$
1+\sum_{n \geq 1} \sum_{\lambda \vdash n}\left(A_{l(\lambda)}(t) \prod_{i=1}^{l(\lambda)}\left[\lambda_{i}\right]_{t}\right) \frac{p_{\lambda}(\mathbf{x})}{z_{\lambda}} z^{n}=\frac{(1-t) H(z)}{H(t z)-t H(z)}
$$

where $\lambda=\left(\lambda_{1} \geq \lambda_{2} \geq \cdots \geq \lambda_{l(\lambda)}\right)$ and

$$
z_{\lambda}:=\prod_{i \geq 1} i^{m_{i}} m_{i} !
$$


if $\lambda$ has $m_{i}$ parts of size $i$ for each $i$. By combining this with (1.5) one obtains the expansion of $\omega W_{n}(\mathbf{x}, t)$ in the power sum symmetric functions given in (1.6).

In this section we derive power sum expansions for the other Smirnov word enumerators. We will use the e-expansion for $\tilde{W}_{n}(\mathbf{x}, t)$ obtained in Section 2 to obtain the following result, which expresses the coefficients of $\frac{p_{\lambda}(\mathbf{x})}{z_{\lambda}}$ in the power sum expansion of $\omega \tilde{W}_{n}(\mathbf{x}, t)$ as a polynomial in $t$ with positive integer coefficients.

Theorem 4.1. For all $n \geq 1$,

$$
\omega \tilde{W}_{n}(\mathbf{x}, t)=\sum_{\lambda \vdash n}\left(A_{l(\lambda)-1}(t) \sum_{i=1}^{l(\lambda)} \lambda_{i} t^{\lambda_{i}} \prod_{j \in[l(\lambda)] \backslash\{i\}}\left[\lambda_{j}\right]_{t}\right) \frac{p_{\lambda}(\mathbf{x})}{z_{\lambda}},
$$

where $\lambda=\left(\lambda_{1} \geq \lambda_{2} \geq \cdots \geq \lambda_{l(\lambda)}\right)$.

We will need the following Lemma, which is implicit in the proof of (4.11) in [35]. We include the proof for the sake of completeness.

Lemma 4.2. For all $k \geq 1$,

$$
\left(\frac{H(z)}{H(t z)}\right)^{k}=1+\sum_{n \geq 1} \sum_{\lambda \vdash n}\left(k^{l(\lambda)} \prod_{i=1}^{l(\lambda)}\left(1-t^{\lambda_{i}}\right)\right) \frac{p_{\lambda}(\mathbf{x})}{z_{\lambda}} z^{n} .
$$

Proof. For each $k \geq 1$, let $\varphi_{k}: \Lambda_{\mathbb{Q}}[z] \rightarrow \Lambda_{\mathbb{Q}}[t, z]$ be the algebra homomorphism determined by

$$
\varphi_{k}\left(p_{r}(\mathbf{x})\right)=k\left(1-t^{r}\right) p_{r}(\mathbf{x})
$$

for all $r \geq 1$. Since $H(z)=1+\sum_{n \geq 1} \sum_{\lambda \vdash n} \frac{p_{\lambda}}{z_{\lambda}} z^{n}$,

$$
\varphi_{k}(H(z))=1+\sum_{n \geq 1} \sum_{\lambda \vdash n}\left(k^{l(\lambda)} \prod_{i=1}^{l(\lambda)}\left(1-t^{\lambda_{i}}\right)\right) \frac{p_{\lambda}}{z_{\lambda}} z^{n} .
$$

To complete the proof we show that $\varphi_{k}(H(z))$ is equal to the left hand side of (4.3). We use the fact that

$$
H(z)=\exp \left(\sum_{r \geq 1} \frac{p_{r}(\mathbf{x})}{r} z^{r}\right)
$$


to obtain

$$
\begin{aligned}
\varphi_{k}(H(z)) & =\exp \left(\sum_{r \geq 1} \frac{\varphi_{k}\left(p_{r}\right)}{r} z^{r}\right) \\
& =\exp \left(\sum_{r \geq 1} \frac{k\left(1-t^{r}\right) p_{r}}{r} z^{r}\right) \\
& =\left(\frac{\exp \left(\sum_{r \geq 1} \frac{p_{r}}{r} z^{r}\right)}{\exp \left(\sum_{r \geq 1} t^{r} \frac{p_{r}}{r} z^{r}\right)}\right)^{k} \\
& =\left(\frac{H(z)}{H(t z)}\right)^{k} .
\end{aligned}
$$

Proof of Theorem 4.1. For each $\lambda \vdash n$, set

$$
c_{\lambda}(t):=A_{l(\lambda)-1}(t) \sum_{i=1}^{l(\lambda)} \lambda_{i} t^{\lambda_{i}} \prod_{j \in[l(\lambda)] \backslash\{i\}}\left[\lambda_{j}\right]_{t} .
$$

We will prove that

$$
\sum_{n \geq 1} \sum_{\lambda \vdash n} c_{\lambda}(t) \frac{p_{\lambda}(\mathbf{x})}{z_{\lambda}} z^{n}=\frac{(1-t) \frac{\partial}{\partial t} H(t z)}{H(t z)-t H(z)},
$$

which by Corollary 2.6 is equivalent to (4.2).

We have

$$
\begin{aligned}
c_{\lambda}(t) & =(t-1) \frac{A_{l(\lambda)-1}(t)}{(t-1)^{l(\lambda)}} \sum_{i=1}^{l(\lambda)} \lambda_{i} t^{\lambda_{i}} \prod_{j \in[l(\lambda)] \backslash\{i\}}\left(t^{\lambda_{j}}-1\right) \\
& =(t-1)(-1)^{l(\lambda)} \frac{A_{l(\lambda)-1}(t)}{(1-t)^{l(\lambda)}} t \frac{d}{d t}\left(\prod_{j=1}^{l(\lambda)}\left(t^{\lambda_{j}}-1\right)\right) \\
& =(t-1) \frac{t A_{l(\lambda)-1}(t)}{(1-t)^{l(\lambda)}} \frac{d}{d t}\left(\prod_{j=1}^{l(\lambda)}\left(1-t^{\lambda_{j}}\right)\right) .
\end{aligned}
$$

For the case $l(\lambda)=1$, we have

$$
c_{(n)}(t)=n t^{n-1} .
$$


For the case $l(\lambda)>1$, we use the classical identity (see [33, Proposition 1.4.4 and equation (1.36)]),

$$
\frac{t A_{m-1}(t)}{(1-t)^{m}}=\sum_{k \geq 1} k^{m-1} t^{k}
$$

for all $m>1$. This yields

$$
\begin{aligned}
c_{\lambda}(t) & =(t-1) \sum_{k \geq 1} k^{l(\lambda)-1} t^{k} \frac{d}{d t}\left(\prod_{j=1}^{l(\lambda)}\left(1-t^{\lambda_{j}}\right)\right) \\
& =(t-1) \sum_{k \geq 1} \frac{t^{k}}{k} \frac{d}{d t}\left(k^{l(\lambda)} \prod_{j=1}^{l(\lambda)}\left(1-t^{\lambda_{j}}\right)\right) .
\end{aligned}
$$

It follows that

$$
\sum_{n \geq 1} \sum_{\lambda \vdash n} c_{\lambda}(t) \frac{p_{\lambda}(\mathbf{x})}{z_{\lambda}} z^{n}=\sum_{n \geq 1} n t^{n-1} \frac{p_{n}(\mathbf{x})}{n} z^{n}+(t-1) \sum_{k \geq 1} \frac{t^{k}}{k} \frac{\partial}{\partial t} U_{k}(\mathbf{x}, t, z)
$$

where

$$
U_{k}(\mathbf{x}, t, z):=\sum_{n \geq 1} \sum_{\substack{\lambda \vdash n \\ l(\lambda)>1}} k^{l(\lambda)} \prod_{j=1}^{l(\lambda)}\left(1-t^{\lambda_{j}}\right) \frac{p_{\lambda}(\mathbf{x})}{z_{\lambda}} z^{n} .
$$

Note that the first summation on the right hand side of (4.7) can be expressed as

$$
\begin{aligned}
\sum_{n \geq 1} n t^{n-1} \frac{p_{n}}{n} z^{n} & =\frac{\partial}{\partial t} \sum_{n \geq 1} t^{n} \frac{p_{n}}{n} z^{n} \\
& =\frac{\partial}{\partial t} \ln H(t z) \\
& =\frac{\frac{\partial}{d t}(H(t z))}{H(t z)}
\end{aligned}
$$

with the second equality following from (4.4).

\footnotetext{
${ }^{4}$ This is Euler's original definition of Eulerian polynomial.
} 
To evaluate the second summation on the right hand side of (4.7), we use Lemma 4.2 to obtain

$$
\begin{aligned}
U_{k}(\mathbf{x}, t, z) & =\sum_{n \geq 1} \sum_{\lambda \vdash n} k^{l(\lambda)} \prod_{j=1}^{l(\lambda)}\left(1-t^{\lambda_{j}}\right) \frac{p_{\lambda}(\mathbf{x})}{z_{\lambda}} z^{n}-\sum_{n \geq 1} k\left(1-t^{n}\right) \frac{p_{n}(\mathbf{x})}{n} z^{n} \\
& =\left(\frac{H(z)}{H(t z)}\right)^{k}-1-k \sum_{n \geq 1}\left(1-t^{n}\right) \frac{p_{n}(\mathbf{x})}{n} z^{n} .
\end{aligned}
$$

By (4.8),

$$
\begin{aligned}
\frac{\partial}{\partial t} U_{k}(\mathbf{x}, t, z) & =k\left(\frac{H(z)}{H(t z)}\right)^{k-1} \frac{\partial}{\partial t}\left(\frac{H(z)}{H(t z)}\right)+k \sum_{n \geq 1} t^{n-1} p_{n}(\mathbf{x}) z^{n} \\
& =-k\left(\left(\frac{H(z)}{H(t z)}\right)^{k} \frac{\frac{\partial}{\partial t}(H(t z))}{H(t z)}-\frac{\frac{\partial}{\partial t}(H(t z))}{H(t z)}\right) \\
& =-k\left(\left(\frac{H(z)}{H(t z)}\right)^{k}-1\right) \frac{\frac{\partial}{\partial t}(H(t z))}{H(t z)} .
\end{aligned}
$$

Hence the second summation is

$$
-\frac{\frac{\partial}{\partial t}(H(t z))}{H(t z)} \sum_{k \geq 1} t^{k}\left(\left(\frac{H(z)}{H(t z)}\right)^{k}-1\right) .
$$

Plugging this and (4.8) into (4.7) yields

$$
\begin{aligned}
\sum_{n \geq 1} \sum_{\lambda \vdash n} c_{\lambda}(t) \frac{p_{\lambda}(\mathbf{x})}{z_{\lambda}} z^{n}= & \frac{\partial}{\partial t}(H(t z)) \\
& +(1-t) \frac{\frac{\partial}{\partial t}(H(t z))}{H(t z)} \sum_{k \geq 1} t^{k}\left(\left(\frac{H(z)}{H(t z)}\right)^{k}-1\right) \\
= & \frac{\frac{\partial}{\partial t}(H(t z))}{H(t z)}\left(1+(1-t) \sum_{k \geq 1} t^{k}\left(\left(\frac{H(z)}{H(t z)}\right)^{k}-1\right)\right) \\
= & \frac{\frac{\partial}{d t}(H(t z))}{H(t z)}\left(1-(1-t) \sum_{k \geq 0} t^{k}+(1-t) \sum_{k \geq 0} t^{k}\left(\frac{H(z)}{H(t z)}\right)^{k}\right) \\
= & \frac{\frac{\partial}{\partial t}(H(t z))}{H(t z)}(1-t) \frac{1}{1-t \frac{H(z)}{H(t z)}} \\
= & \frac{(1-t) \frac{\partial}{\partial t} H(t z)}{H(t z)-t H(z)},
\end{aligned}
$$

which establishes (4.5). 
The following result expresses the coefficients of $\frac{p_{\lambda}(\mathbf{x})}{z_{\lambda}}$ in the power sum expansion of $W_{n}^{<}(\mathbf{x}, t)$ as a polynomial in $t$ with positive integer coefficients.

Theorem 4.3. For all $n \geq 1$,

$$
\omega W_{n}^{<}(\mathbf{x}, t)=\sum_{\lambda \vdash n} \frac{d}{d t}\left(t A_{l(\lambda)-1}(t) \prod_{i=1}^{l(\lambda)}\left[\lambda_{i}\right]_{t}\right) \frac{p_{\lambda}(\mathbf{x})}{z_{\lambda}} .
$$

Proof. Let $c_{\lambda}^{<}(t)$ be the coefficient of $\left(z_{\lambda}\right)^{-1} p_{\lambda}(\mathbf{x})$ in $\omega W_{n}^{<}(\mathbf{x}, t)$. By (1.15) and (1.16) $), \omega W_{n}^{<}(\mathbf{x}, t)=(t-1)^{-1}\left(\omega \tilde{W}_{n}(\mathbf{x}, t)-\omega W_{n}(\mathbf{x}, t)\right)$. Hence from (4.2) and (1.6), we obtain,

$$
\begin{aligned}
c_{\lambda}^{<}(t) & =(t-1)^{-1}\left(A_{l(\lambda)-1}(t) \sum_{i=1}^{l(\lambda)} \lambda_{i} t^{\lambda_{i}} \prod_{j \in[\lambda(l)] \backslash\{i\}}\left[\lambda_{j}\right]_{t}-A_{l(\lambda)}(t) \prod_{i=1}^{l(\lambda)}\left[\lambda_{i}\right]_{t}\right) \\
& =\frac{A_{l(\lambda)-1}(t)}{(t-1)^{l(\lambda)}} \sum_{i=1}^{l(\lambda)} \lambda_{i} t^{\lambda_{i}} \prod_{j \in[\lambda(l)] \backslash\{i\}}\left(t^{\lambda_{j}}-1\right)-\frac{A_{l(\lambda)}(t)}{(t-1)^{l(\lambda)+1}} \prod_{i=1}^{l(\lambda)}\left(t^{\lambda_{i}}-1\right) \\
& =\frac{t A_{l(\lambda)-1}(t)}{(t-1)^{l(\lambda)}} \frac{d}{d t}\left(\prod_{i=1}^{l(\lambda)}\left(t^{\lambda_{i}}-1\right)\right)-\frac{A_{l(\lambda)}(t)}{(t-1)^{l(\lambda)+1}} \prod_{i=1}^{l(\lambda)}\left(t^{\lambda_{i}}-1\right) .
\end{aligned}
$$

From (4.6), one can see that

$$
\frac{d}{d t}\left(\frac{t A_{l(\lambda)-1}(t)}{(1-t)^{l(\lambda)}}\right)=\frac{A_{l(\lambda)}(t)}{(1-t)^{l(\lambda)+1}}
$$

Hence,

$$
\begin{aligned}
c_{\lambda}^{<}(t) & =\frac{t A_{l(\lambda)-1}(t)}{(t-1)^{l(\lambda)}} \frac{d}{d t}\left(\prod_{i=1}^{l(\lambda)}\left(t^{\lambda_{i}}-1\right)\right)+\frac{d}{d t}\left(\frac{t A_{l(\lambda)-1}(t)}{(t-1)^{l(\lambda)}}\right) \prod_{i=1}^{l(\lambda)}\left(t^{\lambda_{i}}-1\right) \\
& =\frac{d}{d t}\left(\frac{t A_{l(\lambda)-1}(t)}{(t-1)^{l(\lambda)}} \prod_{i=1}^{l(\lambda)}\left(t^{\lambda_{i}}-1\right)\right) \\
& =\frac{d}{d t}\left(t A_{l(\lambda)-1}(t) \prod_{i=1}^{l(\lambda)}\left[\lambda_{i}\right]_{t}\right) .
\end{aligned}
$$

Corollary 4.4. For $\lambda \vdash n$, let $c_{\lambda}^{<}(t)$ be the coefficient of $z_{\lambda}^{-1} p_{\lambda}(\mathbf{x})$ in the power sum expansion of $\omega W_{n}^{<}(\mathbf{x}, t)$ and $c_{\lambda}^{>}(t)$ be the coefficient of 
$z_{\lambda}^{-1} p_{\lambda}(\mathbf{x})$ in the power sum expansion of $\omega W_{n}^{>}(\mathbf{x}, t)$. If $\left(a_{0}, a_{1}, \ldots, a_{n-1}\right)$ satisfies

$$
t A_{l(\lambda)-1}(t) \prod_{i=1}^{l(\lambda)}\left[\lambda_{i}\right]_{t}=\sum_{i=0}^{n-1} a_{i} t^{i}
$$

then

$$
c_{\lambda}^{<}(t)=\sum_{i=0}^{n-2}(i+1) a_{i+1} t^{i} \quad \text { and } \quad c_{\lambda}^{>}(t)=\sum_{i=1}^{n-1}(n-i) a_{n-i} t^{i}
$$

Consequently for $\lambda=(n)$,

$$
c_{(n)}^{<}(t)=\sum_{i=0}^{n-2}(i+1) t^{i} \quad \text { and } \quad c_{(n)}^{>}(t)=\sum_{i=1}^{n-1}(n-i) t^{i} .
$$

Proof. We use the fact that $W_{n}^{>}(\mathbf{x}, t)=t^{n-1} W_{n}^{<}\left(\mathbf{x}, t^{-1}\right)$.

One can use Corollary 4.4 to expand the other Smirnov word enumerators in the power sum basis. For instance, one can recover the expansion given in (1.10), which we restate now.

Corollary 4.5 (Ellzey [10]). For $\lambda \vdash n$, let $\tilde{c}_{\lambda}^{\neq}(t)$ be the coefficient of $z_{\lambda}^{-1} p_{\lambda}(\mathbf{x})$ in the power sum expansion of $\omega \tilde{W}_{n}^{\neq}(\mathbf{x}, t)$. Then

$$
\tilde{c}_{\lambda}^{\neq}(t)= \begin{cases}n t A_{l(\lambda)-1}(t) \prod_{i=1}^{l(\lambda)}\left[\lambda_{i}\right]_{t} & \text { if } l(\lambda)>1 \\ n t[n-1]_{t} & \text { if } l(\lambda)=1 .\end{cases}
$$

Proof. We have

$$
\begin{aligned}
\tilde{c}_{\lambda}^{\neq}(t) & =t c_{\lambda}^{<}(t)+c_{\lambda}^{>}(t) \\
& =\sum_{i=1}^{n-1} i a_{i} t^{i}+\sum_{i=1}^{n-1}(n-i) a_{n-i} t^{i},
\end{aligned}
$$

where the $a_{i}$ are as in (4.9).

Now let $l(\lambda)>1$. We claim that $a_{i}=a_{n-i}$ for all $i \in[n-1]$. Indeed, it is well known that the Eulerian polynomials are palindromic and unimodal. Clearly the same is true for each $\left[\lambda_{i}\right]_{t}$. Since the product of palindromic, unimodal polynomials is palindromic, unimodal (see e.g. [29, Proposition B.1]), $\sum_{i=0}^{n-1} a_{i} t^{i}$ is palindromic (and unimodal). Note that $a_{0}=0$ when $l(\lambda)>1$ and $a_{1}, a_{n-1} \neq 0$. Hence the claim holds. It 
follows that

$$
\begin{aligned}
\tilde{c}_{\lambda}^{\neq}(t) & =\sum_{i=1}^{n-1} i a_{i} t^{i}+\sum_{i=1}^{n-1}(n-i) a_{i} t^{i} \\
& =\sum_{i=1}^{n-1} n a_{i} t^{i} \\
& =n t A_{l(\lambda)-1}(t) \prod_{i=1}^{l(\lambda)}\left[\lambda_{i}\right]_{t} .
\end{aligned}
$$

The case $l(\lambda)=1$ follows immediately from (4.10).

For the Smirnov word enumerator $W_{n}^{\neq}(\mathbf{x}, t)$ and the chromatic quasisymmetric function $X_{C_{n}}(\mathbf{x}, t)$, the formulas for the expansion coefficients in the power sum basis that follow from Corollay 4.4 do not seem to reduce to simple formulas except when $\lambda=(n)$. We have the following result in this case.

Corollary 4.6. The coefficient of $n^{-1} p_{n}(\mathbf{x})$ in the power sum expansion of $\omega W_{n}^{\neq}(\mathbf{x}, t)$ is $[n]_{t}+n t[n-2]_{t}$ and in the power sum expansion of $\omega X_{C_{n}}(\mathbf{x}, t)$ is $[2]_{t}[n]_{t}+n t^{2}[n-3]_{t}$.

Remark 4.7. Corollary 4.6 also follows from Corollaries 2.4 and 3.1 since the coefficient of $h_{n}$ in the $h$-expansion of a symmetric function equals the coefficient of $n^{-1} p_{n}$ in the power sum expansion.

\section{EXPANSION IN THE FUNDAMENTAL QUASISYMMETRIC FUNCTIONS}

In [29], Shareshian and the second named author derive, for all labeled incomparability graphs, an expansion of the chromatic quasisymmetic function in Gessel's basis of fundamental quasisymmetric functions, and the first named author does the same for all directed graphs in [10]. The expansion formula in [29, Theorem 3.1] applied to $X_{P_{n}}(\mathbf{x}, t)=W_{n}(\mathbf{x}, t)$ is given in (5.1) below. (A different expansion formula is obtained by applying the formula in [10].) Here we give analogous expansions for $W_{n}^{<}(\mathbf{x}, t), W_{n}^{<}(\mathbf{x}, t)$, and $\omega \tilde{W}_{n}(\mathbf{x}, t)$. These expansions immediately yield expansion formulas for the chromatic quasisymmetric functions $X_{\vec{C}_{n}}(\mathbf{x}, t)$, and $X_{C_{n}}(\mathbf{x}, t)$, which are different from the ones obtained by applying the formula in [10].

For $n \geq 1$ and $S \subseteq[n-1]$, let $D(S)$ be the set of all functions $f:[n] \rightarrow \mathbb{P}$ such that

- $f(i) \geq f(i+1)$ for all $i \in[n-1]$, and

- $f(i)>f(i+1)$ for all $i \in S$. 
The fundamental quasisymmetric function associated with $S \subseteq[n]$ is defined as 5

$$
F_{n, S}(\mathbf{x}):=\sum_{f \in D(S)} x_{f},
$$

where $x_{f}:=x_{f(1)} x_{f(2)} \cdots x_{f(n)}$. In fact, the set $\left\{F_{n, S}: S \subseteq[n-1]\right\}$ is a basis for the vector space of homogeneous quasisymmetric functions of degree $n$ (see [34, Proposition 7.19.1]).

For $\sigma \in \mathfrak{S}_{n}$, define

$$
\operatorname{DES}_{\geq 2}(\sigma):=\{i \in[n-1]: \sigma(i)-\sigma(i+1) \geq 2\}
$$

and

$$
\mathrm{ASC}_{\geq 2}(\sigma):=\{i \in[n-1]: \sigma(i+1)-\sigma(i) \geq 2\}
$$

The expansion formula in [29, Theorem 3.1] applied to $X_{P_{n}}(\mathbf{x}, t)=$ $W_{n}(\mathbf{x}, t)$ yields

$$
\omega W_{n}(\mathbf{x}, t)=\sum_{\sigma \in \mathfrak{S}_{n}} t^{\operatorname{des}(\sigma)} F_{n, \mathrm{DES}_{\geq 2}\left(\sigma^{-1}\right)}(\mathbf{x})
$$

Now we give analogous expansions.

Theorem 5.1. For all $n \geq 1$,

$$
\begin{aligned}
\omega W_{n}^{<}(\mathbf{x}, t) & =\sum_{\substack{\sigma \in \mathfrak{S}_{n} \\
\sigma(1)<\sigma(n)}} t^{\operatorname{des}(\sigma)} F_{n, \mathrm{DES}_{\geq 2}\left(\sigma^{-1}\right)}(\mathbf{x}) \\
\omega W_{n}^{>}(\mathbf{x}, t) & =\sum_{\substack{\sigma \in \mathfrak{S}_{n} \\
\sigma(1)>\sigma(n)}} t^{\operatorname{des}(\sigma)} F_{n, \mathrm{ASC}_{\geq 2}\left(\sigma^{-1}\right)}(\mathbf{x}) .
\end{aligned}
$$

Proof of (5.2). The first part of the proof is similar to that of [29, Theorem 3.1] and [10, Theorem 3.1]. The second part diverges somewhat from these proofs.

Part 1: Given an acyclic orientation $\bar{a}$ of the labeled cycle $C_{n}$, let $E_{\bar{a}}\left(C_{n}\right)$ be the set of directed edges of $C_{n}$ under the orientation $\bar{a}$. Let $A O_{n}^{>}$be the set of acyclic orientations $\bar{a}$ of $C_{n}$ such that $(n, 1) \in$ $E_{\bar{a}}\left(C_{n}\right)$. For each $\bar{a} \in A O_{n}^{>}$, let $W_{\bar{a}} \subseteq W_{n}$ be the set of Smirnov words $w=w_{1} w_{2} \cdots w_{n}$ such that

- $w_{n}<w_{1}$,

- $w_{i}<w_{i+1}$ if $(i, i+1) \in E_{\bar{a}}\left(C_{n}\right)$ and $i \in[n-1]$,

- $w_{i}>w_{i+1}$ if $(i+1, i) \in E_{\bar{a}}\left(C_{n}\right)$ and $i \in[n-1]$.

\footnotetext{
5This is nonstandard notation for Gessel's fundamental quasisymmetric function. Our $F_{n, S}$ is equal to $L_{\alpha(S)}$ in [34, where $\alpha(S)$ is the reverse of the composition associated with $S$.
} 
Let $\operatorname{asc}(\bar{a})$ be the number of edges of $E_{\bar{a}}\left(C_{n}\right)$ of the form $(i, i+1)$ for $i \in[n-1]$. Then by reversing the Smirnov words, we can see that

$$
W_{n}^{<}(\mathbf{x}, t)=\sum_{\substack{w \in W_{n} \\ w_{1}>w_{n}}} x_{w} t^{\operatorname{asc}(w)}=\sum_{\bar{a} \in A O_{n}^{>}} t^{\operatorname{asc}(\bar{a})} \sum_{w \in W_{\bar{a}}} \mathbf{x}_{w},
$$

where $\operatorname{asc}(w):=\left|\left\{i \in[n-1]: w_{i}<w_{i+1}\right\}\right|$.

Now for each acyclic orientation $\bar{a} \in A O_{n}^{>}$, define a poset $P_{\bar{a}}$ on $[n]$ by letting $i<_{P_{\bar{a}}} j$ if $(i, j) \in E_{\bar{a}}\left(C_{n}\right)$ and taking the transitive closure of this relation. Let us define a labeling of $P_{\bar{a}}$ to be a bijection from $P_{\bar{a}}$ to $[n]$. So a labeling is just a permutation in $\mathfrak{S}_{n}$. A labeling $\rho$ is said to be decreasing if $\rho(i)>\rho(j)$ for all $i<_{P_{\bar{a}}} j$. For any labeling $\rho$ of $P_{\bar{a}}$, let $L\left(P_{\bar{a}}, \rho\right)$ be the set of linear extensions of $P_{\bar{a}}$ with the labeling $\rho$.

Now fix a decreasing labeling $\rho_{\bar{a}}$ of $P_{\bar{a}}$ for each $\bar{a} \in A O_{n}^{>}$. For any subset $S \subseteq[n-1]$, define $n-S=\{i \mid n-i \in S\}$. Then by the theory of P-partitions [34, Corollary 7.19.5], we have that

$$
\sum_{w \in W_{\bar{a}}} \mathbf{x}_{w}=\sum_{\sigma \in L\left(P_{\bar{a}}, \rho_{\bar{a}}\right)} F_{n, n-\operatorname{DES}(\sigma)},
$$

where $\operatorname{DES}(\sigma)$ is the usual descent set of a permutation, i.e. $\operatorname{DES}(\sigma)=$ $\{i \in[n-1]: \sigma(i)>\sigma(i+1)\}$.

Let $e: P_{\bar{a}} \rightarrow[n]$ be the identity labeling of $P_{\bar{a}}$, and hence $L\left(P_{\bar{a}}, e\right)$ is the set of linear extensions of $P_{\bar{a}}$ with its original labeling. Note that $\sigma \in L\left(P_{\bar{a}}, e\right)$ if and only if $\rho_{\bar{a}} \sigma \in L\left(P_{\bar{a}}, \rho_{\bar{a}}\right)$, where $\rho_{\bar{a}} \sigma$ denotes the product of $\rho_{\bar{a}}$ and $\sigma$ in $\mathfrak{S}_{n}$. Hence from (5.5), we have

$$
\sum_{w \in W_{\bar{a}}} \mathbf{x}_{w}=\sum_{\sigma \in L\left(P_{\bar{a}}, e\right)} F_{n, n-\operatorname{DES}\left(\rho_{\bar{a}} \sigma\right)} .
$$

Note that if $\sigma \in L\left(P_{\bar{a}}, e\right)$ and $\bar{a} \in A O_{n}^{>}$then $\sigma^{-1}(1)>\sigma^{-1}(n)$. Conversely, every permutation $\sigma \in \mathfrak{S}_{n}$ with $\sigma^{-1}(1)>\sigma^{-1}(n)$ is a linear extension in $L\left(P_{\bar{a}}, e\right)$ for a unique $\bar{a} \in A O_{n}^{>}$. Let $\bar{a}(\sigma)$ denote the unique acyclic orientation of associated with $\sigma$. Now combining this with (5.4) and (5.6) yields,

$$
W_{n}^{<}(\mathbf{x}, t)=\sum_{\substack{\sigma \in \mathfrak{S}_{n} \\ \sigma^{-1}(1)>\sigma^{-1}(n)}} t^{\operatorname{asc}(\bar{a}(\sigma))} F_{n, n-\operatorname{DES}\left(\rho_{\bar{a}(\sigma)} \sigma\right)},
$$

where recall $\rho_{\bar{a}(\sigma)}$ is a decreasing labeling of $P_{\bar{a}(\sigma)}$. Note that $\operatorname{asc}(\bar{a}(\sigma))=$ $\operatorname{des}\left(\left(\sigma^{R}\right)^{-1}\right)$, where $\sigma^{R}$ is the reverse of $\sigma$. Hence

$$
W_{n}^{<}(\mathbf{x}, t)=\sum_{\substack{\sigma \in \mathfrak{S}_{n} \\ \sigma^{-1}(1)>\sigma^{-1}(n)}} t^{\operatorname{des}\left(\left(\sigma^{R}\right)^{-1}\right)} F_{n, n-\operatorname{DES}\left(\rho_{\bar{a}(\sigma)} \sigma\right)} .
$$


Part 2: As in the proof of [29, Theorem 3.1], our next step is to construct a particular decreasing labeling $\tilde{\rho}_{\bar{a}(\sigma)}$ of $P_{\bar{a}(\sigma)}$ for each $\sigma \in$ $L\left(P_{\bar{a}}, e\right)$. However since $C_{n}$ is not the incomparability graph of a poset, the construction used in the proof of [29, Theorem 3.1] does not work in this case. The construction used here is also quite different from that of [10, Theorem 3.1]. Let $p$ be the "smallest" maximal element of $P_{\bar{a}(\sigma)}$ (that is, $p$ is maximal in the poset $P_{\bar{a}(\sigma)}$ and is less than all the other maximal elements in the natural order on $[n])$ and let $\tilde{\rho}_{\bar{a}(\sigma)}(p)=1$. Now remove $p$ from the poset and let $q$ be the smallest maximal element of the remaining poset and let $\tilde{\rho}_{\bar{a}(\sigma)}(q)=2$. Continue this process inductively. It is clear that $\tilde{\rho}_{\bar{a}(\sigma)}$ is a decreasing labeling of $P_{\bar{a}(\sigma)}$.

Claim. If $x$ and $y$ are incomparable in $P:=P_{\bar{a}(\sigma)}$, then $x<y$ implies $\tilde{\rho}_{\bar{a}(\sigma)}(x)<\tilde{\rho}_{\bar{a}(\sigma)}(y)$.

Proof of Claim. One can see this by drawing the Hasse diagram of $P$ minus the edge $(n, 1)$ as a zig-zag path on $[n]$ with the elements of $[n]$ increasing as one moves from left to right. The path consists of up-segments and down-segments. An up-segment is a maximal chain of $P$ of the form $a<_{P} a+1<_{P} \cdots<_{P} a+j$, where $j \geq 1$, and a downsegment is a maximal chain with top and bottom removed unless it's 1 or $\mathrm{n}$, of the form $a>_{P} a+1>_{P} \cdots>_{P} a+j$, where $j \geq 0$. Between any two down-segments there is an up-segment. Let $\alpha_{i}$ be the $i$ th segment from the left for each $i$. One can see that under the labeling $\tilde{\rho}_{\bar{a}(\sigma)}$, the segment $\alpha_{1}$ gets the smallest labels, the segment $\alpha_{2}$ gets the next smallest labels, and so on. Now if $x$ and $y$ are incomparable, they are in different segments $\alpha_{i}$ and $\alpha_{j}$. Clearly if $x<y$ then $i<j$, which implies that $x$ gets a smaller label then $y$. Hence, the claim holds.

Now we show that

$$
\operatorname{DES}\left(\tilde{\rho}_{\bar{a}(\sigma)} \sigma\right)=[n-1] \backslash \operatorname{ASC}_{\geq 2}(\sigma),
$$

for all $\sigma \in \mathfrak{S}_{n}$. If $i \in \operatorname{DES}\left(\tilde{\rho}_{\bar{a}(\sigma)} \sigma\right)$ then $\tilde{\rho}_{\bar{a}(\sigma)} \sigma(i)>\tilde{\rho}_{\bar{a}(\sigma)} \sigma(i+1)$. It thus follows from the claim that if $\sigma(i)$ and $\sigma(i+1)$ are incomparable in $P_{\bar{a}(\sigma)}$ then $\sigma(i)>\sigma(i+1)$, which implies $i \notin \mathrm{ASC}_{\geq 2}(\sigma)$. On the other hand if $\sigma(i)$ and $\sigma(i+1)$ are comparable in $P_{\bar{a}(\sigma)}$ then $\sigma(i+1)$ covers $\sigma(i)$ since $\sigma \in L\left(P_{\bar{a}(\sigma)}, e\right)$. This implies that either $\sigma(i+1)=\sigma(i)+1$ or $\sigma(i+1)=\sigma(i)-1$. In either case, $i \notin \mathrm{ASC}_{\geq 2}(\sigma)$. Thus

$$
\operatorname{DES}\left(\tilde{\rho}_{\bar{a}(\sigma)} \sigma\right) \subseteq[n-1] \backslash \operatorname{ASC}_{\geq 2}(\sigma) .
$$

Conversely, if $i \notin \operatorname{DES}\left(\tilde{\rho}_{\bar{a}(\sigma)} \sigma\right)$ then $\tilde{\rho}_{\bar{a}(\sigma)} \sigma(i)<\tilde{\rho}_{\bar{a}(\sigma)} \sigma(i+1)$. It thus follows from the claim that if $\sigma(i)$ and $\sigma(i+1)$ are incomparable in $P_{\bar{a}(\sigma)}$ then $\sigma(i)<\sigma(i+1)$. Since $j$ and $j+1$ are comparable in $P_{\bar{a}(\sigma)}$ for all $j \in[n-1]$, we have $\sigma(i+1)-\sigma(i) \geq 2$. Thus $i \in \operatorname{ASC}_{\geq 2}(\sigma)$. On the other hand if $\sigma(i)$ and $\sigma(i+1)$ are comparable in $P_{\bar{a}(\sigma)}$ then 
$\sigma(i)<_{P_{\bar{a}(\sigma)}} \sigma(i+1)$ since $\sigma \in L\left(P_{\bar{a}(\sigma)}, e\right)$. But since $\rho$ is a decreasing labeling $\tilde{\rho}_{\bar{a}(\sigma)} \sigma(i)>\tilde{\rho}_{\bar{a}(\sigma)} \sigma(i+1)$, which contradicts our assumption that $i \notin \operatorname{DES}\left(\tilde{\rho}_{\bar{a}(\sigma)} \sigma\right)$. Hence this case is impossible. We have shown

$$
\operatorname{DES}\left(\tilde{\rho}_{\bar{a}(\sigma)} \sigma\right) \supseteq[n-1] \backslash \operatorname{ASC}_{\geq 2}(\sigma),
$$

which completes the proof of (5.8).

Let $\omega$ be the involution on the ring of quasisymmetric functions determined by $\omega F_{n, S}:=F_{n,[n-1] \backslash S}$. Since $\omega$ takes $h_{n}=F_{n, \emptyset}$ to $e_{n}=$ $F_{n,[n-1]}$, the involution $\omega$ restricts to the usual involution on the ring of symmetric functions. Hence by (5.8), equation (5.7) becomes

$$
\begin{aligned}
\omega W_{n}^{<}(\mathbf{x}, t) & =\sum_{\substack{\sigma \in \mathfrak{S}_{n} \\
\sigma^{-1}(1)>\sigma^{-1}(n)}} t^{\operatorname{des}\left(\left(\sigma^{R}\right)^{-1}\right)} F_{n, n-\operatorname{ASC}_{\geq 2}(\sigma)} \\
= & \sum_{\substack{\sigma \in \mathfrak{S}_{n} \\
\sigma^{-1}(1)<\sigma^{-1}(n)}} t^{\operatorname{des}\left(\sigma^{-1}\right)} F_{n, \operatorname{DES}_{\geq 2}(\sigma)} .
\end{aligned}
$$

Remark 5.2. There is an alternative proof of Theorem 5.1 involving standardization, which will be discussed in a forthcoming paper.

Proof of (5.3). A similar proof can be given here. One can also use (5.2) to prove this. Indeed, by the involution on $W_{n}$ which reverses Smirnov words, we obtain

$$
W_{n}^{>}(\mathbf{x}, t)=t^{n-1} W_{n}^{<}\left(\mathbf{x}, t^{-1}\right) .
$$

By the involution on $\mathfrak{S}_{n}$, which reverses permutations,

$$
\sum_{\substack{\sigma \in \mathfrak{S}_{n} \\ \sigma(1)>\sigma(n)}} t^{\operatorname{des}(\sigma)} F_{n, \mathrm{ASC}_{\geq 2}\left(\sigma^{-1}\right)}=\sum_{\substack{\sigma \in \mathfrak{S}_{n} \\ \sigma(1)<\sigma(n)}} t^{n-1-\operatorname{des}(\sigma)} F_{n, \mathrm{DES}_{\geq 2}\left(\sigma^{-1}\right)} .
$$

The result now follows from (5.2).

By combining (5.1), (5.2), and (5.3), one gets fundamental quasisymmetric function expansions of the other Smirnov word enumerators $W_{n}^{=}(\mathbf{x}, t), W_{n}^{\neq}(\mathbf{x}, t), \tilde{W}_{n}(\mathbf{x}, t), \tilde{W}_{n}^{\neq}(\mathbf{x}, t)$ and of the chromatic quasisymmetric function $X_{C_{n}}(\mathbf{x}, t)$. The resulting expansion for $\tilde{W}_{n}(\mathbf{x}, t)$ has a particularly nice form.

Corollary 5.3. For all $n \geq 1$,

$$
\omega \tilde{W}_{n}(\mathbf{x}, t)=\sum_{\sigma \in \mathfrak{S}_{n}} t^{\operatorname{cdes}(\sigma)} F_{n, \mathrm{DES}_{\geq 2}\left(\sigma^{-1}\right)}(\mathbf{x})
$$


Proof. We use the fact that $\tilde{W}_{n}(\mathbf{x}, t)=t W_{n}^{<}(\mathbf{x}, t)+\left(W_{n}(\mathbf{x}, t)-W_{n}^{<}(\mathbf{x}, t)\right)$. By (5.1) and (5.2),

$$
\omega W_{n}(\mathbf{x}, t)-\omega W_{n}^{<}(\mathbf{x}, t)=\sum_{\substack{\sigma \in \mathfrak{S}_{n} \\ \sigma(1)>\sigma(n)}} t^{\operatorname{des}(\sigma)} F_{n, \mathrm{DES}_{\geq 2}\left(\sigma^{-1}\right)} .
$$

It follows from this and (5.2) that

$$
\begin{aligned}
\omega \tilde{W}_{n}(\mathbf{x}, t) & =\sum_{\substack{\sigma \in \mathfrak{S}_{n} \\
\sigma(1)<\sigma(n)}} t^{\operatorname{des}(\sigma)+1} F_{n, \mathrm{DES}_{\geq 2}\left(\sigma^{-1}\right)}+\sum_{\substack{\sigma \in \mathfrak{S}_{n} \\
\sigma(1)>\sigma(n)}} t^{\operatorname{des}(\sigma)} F_{n, \mathrm{DES}_{\geq 2}\left(\sigma^{-1}\right)} \\
& =\sum_{\sigma \in \mathfrak{S}_{n}} t^{\operatorname{cdes}(\sigma)} F_{n, \mathrm{DES}_{\geq 2}\left(\sigma^{-1}\right)} .
\end{aligned}
$$

There are various ways to specialize expansions in the fundamental quasisymmetric functions to obtain enumerative results. One way is by setting $x_{i}=1$ if $i \in[m]$ and $x_{i}=0$ otherwise, in a formal power series $f(\mathbf{x})$. We denote this specialization by $f\left(1^{m}\right)$. (Another way is discussed in the next section.) It is not difficult to show that (see [34, Section 7.19]),

$$
F_{n, S}\left(1^{m}\right)=\left(\begin{array}{c}
m+n-1-|S| \\
n
\end{array}\right),
$$

for all $S \subseteq[n-1]$. It is clear that

$$
W_{n}\left(1^{m}, t\right)=\sum_{w \in W_{n} \cap[m]^{n}} t^{\operatorname{des}(w)} .
$$

Hence by (5.1) and the fact that $\omega F_{n, S}=F_{n,[n-1] \backslash S}$,

$$
\sum_{w \in W_{n} \cap[m]^{n}} t^{\operatorname{des}(w)}=\sum_{\sigma \in \mathfrak{S}_{n}} t^{\operatorname{des}(\sigma)}\left(\begin{array}{c}
m+\left|\mathrm{DES}_{\geq 2}\left(\sigma^{-1}\right)\right| \\
n
\end{array}\right),
$$

for all $m, n \in \mathbb{P}$. Analogous formulas can be obtained by applying the same specialization to the expansions (5.2), (5.3), and (5.9). The expansions (5.2) and (5.9) yield the following result.

Corollary 5.4. For all $m, n \geq 1$,

$$
\sum_{\substack{w \in W_{n} \cap[m]^{n} \\
w_{1}<w_{n}}} t^{\operatorname{des}(w)}=\sum_{\substack{\sigma \in \mathfrak{S}_{n} \\
\sigma(1)<\sigma(n)}} t^{\operatorname{des}(\sigma)}\left(\begin{array}{c}
m+\left|\operatorname{DES}_{\geq 2}\left(\sigma^{-1}\right)\right| \\
n
\end{array}\right)
$$

and

$$
\sum_{w \in W_{n} \cap[m]^{n}} t^{\operatorname{cdes}(w)}=\sum_{\sigma \in \mathfrak{S}_{n}} t^{\operatorname{cdes}(\sigma)}\left(\begin{array}{c}
m+\left|\mathrm{DES}_{\geq 2}\left(\sigma^{-1}\right)\right| \\
n
\end{array}\right) .
$$


Remark 5.5. In [21, 22], LoBue Tiefenbruck and Remmel study the distribution of a pair of interesting statistics on Smirnov words in $[\mathrm{m}]^{n}$ different from des and cdes. They use the fact that their statistics are preserved by a contraction map from unconstrained words to Smirnov words to transfer their results from Smirnov words to unconstrained words. Since des and cdes are also preserved by the contraction map, we can also transfer our results to unconstrained words.

Remark 5.6. We now describe a proof of (1.4) that is different from the proof in [26] discussed in the introduction. Theorem 3.1 of [29] gives a fundamental quasisymmetric function expansion of the chromatic quasisymmetric function $X_{G}(\mathbf{x}, t)$ when $G$ is an incomparability graph. (This reduces to (5.1) when $G$ is the path $P_{n}$.) In [2], Athanasiadis proves that the fundamental quasisymmetric function expansion implies the conjectured formula (7.14) of [29], which gives a power sum symmetric function expansion of $X_{G}(\mathbf{x}, t)$ when $G$ is a natural unit interval graph. It is shown in [29, Proof of Proposition 7.9] that when $G=P_{n}$, the power sum symmetric function expansion reduces to (7.15) of [29], which is

$$
\omega X_{P_{n}}(\mathbf{x}, t)=\sum_{\lambda \vdash n}\left(A_{l(\lambda)}(t) \prod_{i=1}^{l(\lambda)}\left[\lambda_{i}\right]_{t}\right) \frac{p_{\lambda}(\mathbf{x})}{z_{\lambda}} .
$$

Hence since $W_{n}(\mathbf{x}, t)=X_{P_{n}}(\mathbf{x}, t)$, Stembridge's formula (4.1) implies (1.5), which is equivalent to (1.4).

\section{VARiations of $q$-EULERIAN POLYNOMials}

Recall that the Eulerian polynomials $A_{n}(t)$ have two well-known combinatorial interpretations, which are given by

$$
A_{n}(t):=\sum_{\sigma \in \mathfrak{S}_{n}} t^{\operatorname{des}(\sigma)}=\sum_{\sigma \in \mathfrak{S}_{n}} t^{\operatorname{exc}(\sigma)}
$$

and that Euler's exponential generating function for the Eulerian polynomials is given by

$$
1+\sum_{n \geq 1} A_{n}(t) \frac{z^{n}}{n !}=\frac{(1-t) e^{z}}{e^{t z}-t e^{z}}
$$

In [26] and [29], Shareshian and the second named author obtained combinatorial interpretations of the $q$-Eulerian polynomials $A_{n}(q, t)$ 
that satisfy the $q$-exponential generating function formula

$$
\begin{aligned}
1+\sum_{n \geq 1} A_{n}(q, t) \frac{z^{n}}{[n]_{q} !} & =\frac{(1-t) \exp _{q}(z)}{\exp _{q}(t z)-t \exp _{q}(z)} \\
& =1+\frac{(1-t) \sum_{i \geq 2}[i]_{t} \frac{z^{i}}{[i]_{q} !}}{\exp _{q}(t z)-t \exp _{q}(z)}
\end{aligned}
$$

The interpretation in [26] is given by

$$
A_{n}^{\mathrm{maj}, \operatorname{exc}}(q, t)=\sum_{\sigma \in \mathfrak{S}_{n}} q^{\mathrm{maj}(\sigma)-\operatorname{exc}(\sigma)} t^{\operatorname{exc}(\sigma)}
$$

and the interpretation in [29] is given by

$$
A_{n}(q, t)=\sum_{\sigma \in \mathfrak{S}_{n}} q^{\mathrm{maj}_{\geq 2}\left(\sigma^{-1}\right)} t^{\operatorname{des}(\sigma)}
$$

where

$$
\operatorname{maj}(\sigma)=\sum_{\substack{i \in[n-1] \\ \sigma(i+1)>\sigma(i)}} i \text { and } \operatorname{maj}_{\geq 2}(\sigma):=\sum_{\substack{i \in[n-1] \\ \sigma(i+1)-\sigma(i) \geq 2}} i
$$

Both $q$-analogs of $A_{n}(t)$ were obtained by expanding $\omega W_{n}(\mathbf{x}, t)$ in the fundamental quasisymmetric functions and then taking the stable principal specialization. A formulation of the expansion obtained in [26] yields (6.2), while the formulation (5.1) obtained in [29] yields (6.3); see [29, Proof of Theorem 9.7]. From this it follows that the two q-analogs are equal. (A subsequent bijective proof was obtained in [5].)

In this section, we use results of the previous sections to obtain analogs of (6.1) for variations of the interpretation of $A_{n}(q, t)$ given by (6.3). The variations are defined by

$$
A_{n}^{<}(q, t):=\sum_{\substack{\sigma \in \mathfrak{S}_{n} \\ \sigma(1)<\sigma(n)}} q^{\mathrm{maj}_{\geq 2}\left(\sigma^{-1}\right)} t^{\operatorname{des}(\sigma)}
$$

and

$$
\tilde{A}_{n}(q, t):=\sum_{\sigma \in \mathfrak{S}_{n}} q^{\mathrm{maj}_{\geq 2}\left(\sigma^{-1}\right)} t^{\operatorname{cdes}(\sigma)}
$$

We also obtain nice formulas for $A_{n}^{<}(q, t)$ and $\tilde{A}_{n}(q, t)$ evaluated at $n$th roots of unity.

The stable principal specialization $\operatorname{ps}(G(\mathbf{x}))$ of a quasisymmetric function $G(\mathbf{x})$ is obtained from $G(\mathbf{x})$ by substituting $q^{i-1}$ for $x_{i}$ for all $i \geq 1$. By [14, Lemma 5.2],

$$
\operatorname{ps}\left(F_{n, S}(x)\right)=\frac{\sum_{i \in S} q^{i}}{(1-q)\left(1-q^{2}\right) \cdots\left(1-q^{n}\right)}
$$


for all $S \subseteq[n-1]$. Hence by (5.1), (5.2), and (5.9), respectively,

$$
\begin{aligned}
& \operatorname{ps}\left(\omega W_{n}(\mathbf{x}, t)\right)=\frac{A_{n}(q, t)}{(1-q)\left(1-q^{2}\right) \cdots\left(1-q^{n}\right)} \\
& \operatorname{ps}\left(\omega W_{n}^{<}(\mathbf{x}, t)\right)=\frac{A_{n}^{<}(q, t)}{(1-q)\left(1-q^{2}\right) \cdots\left(1-q^{n}\right)} \\
& \operatorname{ps}\left(\omega \tilde{W}_{n}(\mathbf{x}, t)\right)=\frac{\tilde{A}_{n}(q, t)}{(1-q)\left(1-q^{2}\right) \cdots\left(1-q^{n}\right)} .
\end{aligned}
$$

In [26, 29], first $\omega$ is applied to both sides of (1.5), then the stable principal specialization is taken using (6.4), and finally $z$ is replaced by $(1-q) z$ resulting in (6.1). By doing the same to (2.12) and (2.16), using (6.5) and (6.6), respectively, we obtain the following result.

Theorem 6.1. We have

$$
\begin{aligned}
& \sum_{n \geq 1} A_{n}^{<}(q, t) \frac{z^{n}}{[n]_{q} !}=\frac{(1-t) \frac{\partial}{\partial t} \sum_{i \geq 2}[i]_{t} \frac{z^{i}}{[i]_{q} !}}{\exp _{q}(t z)-t \exp _{q}(z)} \\
& \sum_{n \geq 1} \tilde{A}_{n}(q, t) \frac{z^{n}}{[n]_{q} !}=\frac{(1-t) \frac{\partial}{\partial t} \exp _{q}(t z)}{\exp _{q}(t z)-t \exp _{q}(z)} .
\end{aligned}
$$

In [24, Corollary 6.2], Sagan, Shareshian and the second named author show that for every $n$th root of unity $\xi$, the coefficients of the polynomial $A_{n}(\xi, t)$ are positive integers. More precisely, they show that if $k \mid n$ and $\xi_{k}$ is any primitive $k$ th root of unity then

$$
A_{n}\left(\xi_{k}, t\right)=A_{\frac{n}{k}}(t)[k]_{t}^{\frac{n}{k}} .
$$

Consequently, $A_{n}\left(\xi_{k}, t\right)$ is a palindromic, unimodal polynomial in $\mathbb{N}[t]$. Here we prove analogous results for other Smirnov word enumerators.

A key tool in the proof of (6.9) is the following result, which is implicit in [7] and stated explicitly in [24].

Lemma 6.2 (see [24, Proposition 3.1]). Let $R$ be a commutative ring. Suppose $u(q) \in R[q]$ and there exists a homogeneous symmetric function $U(\mathbf{x})$ of degree $n$ with coefficients in $R$ such that

$$
u(q)=(1-q)\left(1-q^{2}\right) \ldots\left(1-q^{n}\right) \operatorname{ps}(U(\mathbf{x})) .
$$

If $k \mid n$ then $u\left(\xi_{k}\right)$ is the coefficient of $z_{\left(k \frac{n}{k}\right)}^{-1} p_{\left(k \frac{n}{k}\right)}$ in the expansion of $U(\mathbf{x})$ in the power sum basis. 
In [24], (6.9) is proved by setting $R=\mathbb{Q}[t]$ and $U(\mathbf{x})=\omega W_{n}(\mathbf{x}, t)$ in Lemma 6.2. By (6.4), $u(q)=A_{n}(q, t)$. Hence it follows from Lemma 6.2 that $A_{n}\left(\xi_{k}, t\right)$ equals the coefficient of $z_{\left(k^{\frac{n}{k}}\right)}^{-1} p_{\left(k^{\frac{n}{k}}\right)}$ in the expansion of $\omega W_{n}(\mathbf{x}, t)$ in the power sum basis, which by (1.6) equals $A_{\frac{n}{k}}(t)[k]_{t}^{\frac{n}{k}}$. We use a similar argument to obtain the following result. Indeed, to prove (6.10) below, we set $U(\mathbf{x})=\omega W_{n}^{<}(\mathbf{x}, t)$ and use (6.5) and Theorem 4.3. To prove (6.11) below, we set $U(\mathbf{x})=\omega \tilde{W}_{n}(\mathbf{x}, t)$ and use (6.6) and Theorem 4.1.

Theorem 6.3. Let $n \geq 2$ and $k \mid n$. If $\xi_{k}$ is any primitive $k$ th root of unity then

$$
A_{n}^{<}\left(\xi_{k}, t\right)=\frac{d}{d t}\left(t A_{\frac{n}{k}-1}(t)[k]_{t}^{\frac{n}{k}}\right)
$$

and

$$
\tilde{A}_{n}\left(\xi_{k}, t\right)=n t^{k} A_{\frac{n}{k}-1}(t)[k]_{t}^{\frac{n}{k}-1} .
$$

Consequently, $A_{n}^{<}\left(\xi_{k}, t\right), \tilde{A}_{n}\left(\xi_{k}, t\right) \in \mathbb{N}[t]$ and $\tilde{A}_{n}\left(\xi_{k}, t\right)$ is palindromic and unimodal.

Corollary 6.4. For all $n \geq 2$,

$$
A_{n}^{<}(1, t)=\frac{d}{d t}\left(t A_{n-1}(t)\right)
$$

and

$$
\tilde{A}_{n}(1, t)=n t A_{n-1}(t) .
$$

Equations (6.12) and (6.13) have elementary bijective proofs. Indeed, for each $\sigma \in \mathfrak{S}_{n}$, such that $\sigma(n)=n$, let $\mathcal{C}_{\sigma}$ be the set of circular rearrangements of $\sigma$. Clearly, $\left|\mathcal{C}_{\sigma}\right|=n$ and for each $\tau \in \mathcal{C}_{\sigma}$, we have $\operatorname{cdes}(\tau)=\operatorname{des}(\sigma)+1$. Hence,

$$
\begin{aligned}
\tilde{A}_{n}(1, t) & =\sum_{\substack{\sigma \in \mathfrak{S}_{n} \\
\sigma(n)=n}} \sum_{\tau \in \mathcal{C}_{\sigma}} t^{\operatorname{cdes}(\tau)} \\
& =\sum_{\substack{\sigma \in \mathfrak{S}_{n} \\
\sigma(n)=n}} n t^{\operatorname{des}(\sigma)+1} \\
& =n \sum_{\substack{\sigma \in \mathfrak{S}_{n-1}\\
}} t^{\operatorname{des}(\sigma)+1} \\
& =n t A_{n-1}(t) .
\end{aligned}
$$


Now for each $\sigma \in \mathfrak{S}_{n}$, such that $\sigma(n)=n$, let

$$
\mathcal{C}_{\sigma}^{<}:=\left\{\tau \in \mathcal{C}_{\sigma}: \tau(1)<\tau(n)\right\} .
$$

Clearly, $\left|\mathcal{C}_{\sigma}^{<}\right|=\operatorname{des}(\sigma)+1$ and for each $\tau \in \mathcal{C}_{\sigma}^{<}$, we have $\operatorname{des}(\tau)=\operatorname{des}(\sigma)$. Hence

$$
\begin{aligned}
A_{n}^{<}(1, t) & =\sum_{\substack{\sigma \in \mathfrak{S}_{n} \\
\sigma(n)=n}} \sum_{\tau \in \mathcal{C}_{\sigma}^{<}} t^{\operatorname{des}(\tau)} \\
& =\sum_{\substack{\sigma \in \mathfrak{S}_{n} \\
\sigma(n)=n}}(\operatorname{des}(\sigma)+1) t^{\operatorname{des}(\sigma)} \\
& =\sum_{\substack{\sigma \in \mathfrak{S}_{n-1}\\
}}(\operatorname{des}(\sigma)+1) t^{\operatorname{des}(\sigma)} \\
& =\frac{d}{d t}\left(t A_{n-1}(t)\right) .
\end{aligned}
$$

By combining (6.9) with (6.10) and with (6.11), we obtain the following generalization of the previous corollary.

Corollary 6.5. Let $n \geq 2$ and $k \mid n$. If $\xi_{k}$ is any primitive $k$ th root of unity then

and

$$
A_{n}^{<}\left(\xi_{k}, t\right)=\frac{d}{d t}\left(t[k]_{t} A_{n-k}\left(\xi_{k}, t\right)\right)
$$

$$
\tilde{A}_{n}\left(\xi_{k}, t\right)=n t^{k} A_{n-k}\left(\xi_{k}, t\right)
$$

\section{REFERENCES}

[1] P. Alexandersson and G. Panova, LLT polynomials, chromatic quasisymmetric functions and graphs with cycles, preprint, arXiv:1705.10353.

[2] C. Athanasiadis, Power sum expansion of chromatic quasisymmetric functions, Electron. J. Combin. 22 (2015), P 2.7, 9pp.

[3] C. Athanasiadis, A survey of subdivisions and local h-vectors, in The Mathematical Legacy of Richard P. Stanley (P. Hersh, T. Lam, P. Pylyavskyy and V. Reiner, eds.), 39-51, Amer. Math. Society, Providence, RI, 2016.

[4] P. Brosnan and T.Y. Chow, Unit interval orders and the dot action on the cohomology of regular semisimple Hessenberg varieties, Adv. Math. 329 (2018), 955-1001.

[5] A. Bigeni, A new bijection relating q-Eulerian polynomials, Adv. Applied Math. 81 (2016), 212-239.

[6] L. Carlitz, R. Scoville, and T. Vaughan, Enumeration of pairs of sequences by rises, falls and levels, Manuscripta Math. 19 (1976), 211-243.

[7] J. Désarménien, Fonctions symétriques associées à des suites classiques de nombres, Ann. Scient. Éc. Norm. Sup. t. 16, 1983, 271-304.

[8] J. Dollhopf, I. Goulden, and C. Greene, Words avoiding a reflexive acyclic relation, Electon. J. Combin. 11 (2006), \#R28. 
[9] B. Ellzey, A directed graph generalization of chromatic quasisymmetric functions, Séminaire Lotharingien de Combinatoire, Proceedings of the 29th Conference on Formal Power Series and Algebraic Combinatorics 78B (2017), Article \#74, 12 pp.

[10] B. Ellzey, Chromatic quasisymmetric functions of directed graphs, preprint, arXiv:1709.00454.

[11] B. Ellzey, On Chromatic Quasisymmetric Functions of Directed Graphs, University of Miami Ph.D. dissertation (2018), Open Access Dissertations, 2091. https://scholarlyrepository.miami.edu/oa_dissertations/2091.

[12] P. Flajolet and R. Sedgewick, Analytic Combinatorics, Cambridge University Press, Cambridge, 2009.

[13] U. Freiberg, C. Heuberger, and H. Prodinger, Application of Smirnov words to waiting time distributions of runs, Electron. J. Combin. 24 (3) (2017), \#P3.55.

[14] I.M. Gessel and C. Reutenauer, Counting permutations with given cycle structure and descent set, J. Combin. Th. A 64 (1993), 189-215.

[15] I.P. Goulden and D. M. Jackson, Combinatorial Enumeration, Wiley, New York, 1983.

[16] D. Grinberg and V. Reiner, Hopf Algebras in Combinatorics, preprint, arXiv:1409.8356

[17] M. Guay-Paquet, A modular relation for the chromatic symmetric functions of (3+1)-free posets, preprint, arXiv:1306.2400

[18] M. Guay-Paquet, A second proof of the Shareshian-Wachs conjecture, by way of a new Hopf algebra, preprint, arXiv:1601.05498.

[19] A. Henderson and M.L. Wachs, Unimodality of Eulerian quasisymmetric functions, J. Combin. Th. A 119 (2012), 135-145.

[20] S. Linusson, J. Shareshian, and M.L. Wachs, Rees products and lexicographic shellability, J. Combin. 3 (2012), 243-276.

[21] J. LoBue Tiefenbruck and J.B. Remmel, The $\mu$ pattern in words, J. Combin. 5 (2014), 379-417.

[22] J. LoBue Tiefenbruck and J.B. Remmel, Q-analogues of convolutions of Fibonacci numbers, Australas. J. Combin. 64 (2016), 166-193.

[23] A. Matveev, Pattern recognition on oriented matroids: symmetric cycles in the hypercube graphs. III, preprint, arXiv:1805.06810.

[24] B. Sagan, J. Shareshian, and M.L. Wachs, Eulerian quasisymmetric functions and cyclic sieving, Advances in Applied Math. 46 (2011), 536-562.

[25] J. Shareshian and M.L. Wachs, q-Eulerian polynomials: excedance number and major index, Electron. Res. Announc. Amer. Math. Soc. 13 (2007), $33-45$.

[26] J. Shareshian and M.L. Wachs, Eulerian quasisymmetric functions, Advances in Math. 225 (2010), 2921-2966.

[27] J. Shareshian and M.L. Wachs, Poset homology of Rees products and qEulerian polynomials, Electron. J. Combin. 16 (2009), R20, 29 pp.

[28] J. Shareshian and M.L. Wachs, Chromatic quasisymmetric functions and Hessenberg varieties, in Configuration Spaces, Proceedings (A. Bjorner, F. Cohen, C. De Concini, C. Procesi and M. Salvetti, eds.), 433-460, Edizioni della Normale, Pisa, 2012. 
[29] J. Shareshian and M.L. Wachs, Chromatic quasisymmetric functions, Advances in Math. 295 (2016), 497-551.

[30] J. Shareshian and M.L. Wachs, From poset topology to q-Eulerian polynomials to Stanley's chromatic symmetric functions, in The Mathematical Legacy of Richard P. Stanley (P. Hersh, T. Lam, P. Pylyavskyy and V. Reiner, eds.), 301-321, Amer. Math. Society, Providence, RI, 2016.

[31] R.P. Stanley, Log-concave and unimodal sequences in algebra, combinatorics, and geometry, in Graph theory and its applications: East and West (Jinan, 1986), 500-535, Ann. New York Acad. Sci., 576, New York Acad. Sci., New York, 1989.

[32] R.P. Stanley, A symmetric function generalization of the chromatic polynomial of a graph, Advances in Math. 111 (1995), 166-194.

[33] R.P. Stanley, Enumerative combinatorics, Vol. 1, 2nd ed., Cambridge Studies in Advanced Mathematics, 49, Cambridge University Press, Cambridge, 1997.

[34] R.P. Stanley, Enumerative combinatorics, Vol. 2, Cambridge Studies in Advanced Mathematics, 62, Cambridge University Press, Cambridge, 1999.

[35] J.R. Stembridge, Eulerian numbers, tableaux, and the Betti numbers of a toric variety, Discrete Math. 99 (1992), 307-320.

Department of Mathematics, University of Miami, Coral Gables, FL 33124

E-mail address: ellzey@math.miami.edu

Department of Mathematics, University of Miami, Coral Gables, FL 33124

E-mail address: wachs@math.miami.edu 